\title{
Determining the neutrino mass ordering and oscillation parameters with KM3NeT/ORCA
}

\section{KM3NeT Collaboration}

S. Aiello ${ }^{1}$, A. Albert ${ }^{2,55}$, S. Alves Garre ${ }^{3}$, Z. Aly ${ }^{4}$, A. Ambrosone ${ }^{5,6}$, F. Ameli ${ }^{7}$, M. Andre ${ }^{8}$, G. Androulakis ${ }^{9}$, M. Anghinolfi ${ }^{10}$, M. Anguita ${ }^{11}$, G. Anton ${ }^{12}$, M. Ardid ${ }^{13}$, S. Ardid ${ }^{13}$, J. Aublin ${ }^{14}$, C. Bagatelas ${ }^{9}$, B. Baret ${ }^{14}$, S. Basegmez du Pree ${ }^{15}$, M. Bendahman ${ }^{16}$, F. Benfenati ${ }^{17,18}$, E. Berbee ${ }^{15}$, A. M. van den Berg ${ }^{19}$, V. Bertin ${ }^{4}$, S. Biagi ${ }^{20}$, M. Bissinger ${ }^{12}$, M. Boettcher ${ }^{21}$, M. Bou Cabo ${ }^{22}$, J. Boumaaza ${ }^{16}$, M. Bouta ${ }^{23}$, M. Bouwhuis ${ }^{15}$, C. Bozza ${ }^{24}$, H. Brânzaş ${ }^{25}$, R. Bruijn ${ }^{15,26}$, J. Brunner ${ }^{4}$, R. Bruno ${ }^{1}$, E. Buis ${ }^{27}$, R. Buompane ${ }^{5,28}$, J. Busto ${ }^{4}$, B. Caiffi ${ }^{10}$, D. Calvo ${ }^{3}$, A. Capone $^{7,29}$, V. Carretero ${ }^{3}$, P. Castaldi ${ }^{17,30}$, S. Celli ${ }^{7,29}$, M. Chabab ${ }^{31}$, N. Chau ${ }^{14}$, A. Chen ${ }^{32}$, S. Cherubini ${ }^{20,33}$, V. Chiarella ${ }^{34}$, T. Chiarusi ${ }^{17}$, M. Circella ${ }^{35}$, R. Cocimano ${ }^{20}$, J. A. B. Coelho ${ }^{14}$, A. Coleiro ${ }^{14}$, M. Colomer Molla ${ }^{3,14}$, R. Coniglione ${ }^{20}$, P. Coyle ${ }^{4}$, A. Creusot ${ }^{14}$, A. Cruz ${ }^{36}$, G. Cuttone ${ }^{20}$, R. Dallier ${ }^{37}$, B. De Martino ${ }^{4}$, M. De Palma ${ }^{35,38}$, M. Di Marino ${ }^{39}$, I. Di Palma ${ }^{7,29}$, A. F. Díaz ${ }^{11}$, D. Diego-Tortosa ${ }^{13}$, C. Distefano ${ }^{20}$, A. Domi ${ }^{10,40}$, C. Donzaud $^{14}$, D. Dornic ${ }^{4}$, M. Dörr ${ }^{41}$, D. Drouhin ${ }^{2,55}$, T. Eberl ${ }^{12}$, A. Eddyamoui ${ }^{16}$, T. van Eeden ${ }^{15}$, D. van Eijk ${ }^{15}$, I. El Bojaddaini ${ }^{23}$, D. Elsaesser ${ }^{41}$, A. Enzenhöfer ${ }^{4}$, V. Espinosa ${ }^{13}$, P. Fermani ${ }^{7,29}$, G. Ferrara ${ }^{20,33}$, M. D. Filipović ${ }^{42}$, F. Filippini ${ }^{17,18}$, L. A. Fusco ${ }^{4}$, T. Gal ${ }^{12}$, A. Garcia Soto ${ }^{15}$, F. Garufi ${ }^{5,6}$, Y. Gatelet ${ }^{14}$, N. Geißelbrecht ${ }^{12}$, L. Gialanella ${ }^{5,28}$, E. Giorgio ${ }^{20}$, S. R. Gozzini ${ }^{7,44}$, R. Gracia ${ }^{15}$, K. Graf ${ }^{12}$, D. Grasso ${ }^{56}$, G. Grella ${ }^{39}$, D. Guderian ${ }^{57}$, C. Guidi ${ }^{10,40}$, J. Haefner ${ }^{12}$, S. Hallmann ${ }^{12, \text { a }, \text { H. Hamdaoui }}{ }^{16}$, H. van Haren ${ }^{45}$, A. Heijboer ${ }^{15}$, A. Hekalo ${ }^{41}$, L. Hennig ${ }^{12}$, J. J. Hernández-Rey ${ }^{3}$, J. Hofestädt ${ }^{12, b}$, F. Huang ${ }^{4}$, W. Idrissi Ibnsalih ${ }^{5,28}$, G. Illuminati ${ }^{3,14}$, C. W. James ${ }^{36}$, M. de Jong ${ }^{15}$, P. de Jong ${ }^{15,26}$, B. J. Jung ${ }^{15}$, M. Kadler ${ }^{41}$, P. Kalaczyński ${ }^{46}$, O. Kalekin ${ }^{12}$, U. F. Katz ${ }^{12}$, N. R. Khan Chowdhury ${ }^{3}$, G. Kistauri ${ }^{47}$, F. van der Knaap ${ }^{27}$, P. Kooijman ${ }^{26,58}$, A. Kouchner ${ }^{14,48}$, M. Kreter ${ }^{21}$, V. Kulikovskiy ${ }^{10}$, R. Lahmann ${ }^{12}$, M. Lamoureux ${ }^{14}$, G. Larosa ${ }^{20}$, C. Lastoria ${ }^{4}$, R. Le Breton ${ }^{14}$, S. Le Stum $^{4}$, O. Leonardi ${ }^{20}$, F. Leone ${ }^{20,33}$, E. Leonora ${ }^{1}$, N. Lessing ${ }^{12}$, G. Levi ${ }^{17,18}$, M. Lincetto ${ }^{4}$, M. Lindsey Clark ${ }^{14}$, T. Lipreau $^{37}$, F. Longhitano ${ }^{1}$, D. Lopez-Coto ${ }^{49}$, L. Maderer ${ }^{14}$, J. Mańczak ${ }^{3}$, K. Mannheim ${ }^{41}$, A. Margiotta ${ }^{17,18}$, A. Marinelli ${ }^{5}$, C. Markou' ${ }^{9}$ L. Martin ${ }^{37}$, J. A. Martínez-Mora ${ }^{13}$, A. Martini ${ }^{34}$, F. Marzaioli ${ }^{5,28}$, S. Mastroianni ${ }^{5}$, K. W. Melis ${ }^{15}$, G. Miele ${ }^{5,6}$, P. Migliozzi ${ }^{5}$, E. Migneco ${ }^{20}$, P. Mijakowski ${ }^{46}$, L. S. Miranda ${ }^{50}$, C. M. Mollo ${ }^{5}$, M. Morganti ${ }^{56,59}$, M. Moser $^{12}$, A. Moussa ${ }^{23}$, R. Muller ${ }^{15}$, M. Musumeci ${ }^{20}$, L. Nauta ${ }^{15}$, S. Navas ${ }^{49}$, C. A. Nicolau $^{7}$, B. Ó. Fearraigh ${ }^{15,26}$, M. O’Sullivan ${ }^{36}$, M. Organokov ${ }^{2}$, A. Orlando ${ }^{20}$, J. Palacios González ${ }^{3}$, G. Papalashvili ${ }^{47}$, R. Papaleo $^{20}$, C. Pastore ${ }^{35}$, A. M. Păun ${ }^{25}$, G. E. Păvălaş ${ }^{25}$, C. Pellegrino ${ }^{18,60}$, M. Perrin-Terrin ${ }^{4, c}$ (D),

V. Pestel ${ }^{15}$, P. Piattelli ${ }^{20}$, C. Pieterse ${ }^{3}$, K. Pikounis ${ }^{9}$, O. Pisanti ${ }^{5,6}$, C. Poirè ${ }^{13}$, V. Popa ${ }^{25}$, T. Pradier ${ }^{2}$, G. Pühlhofer $^{51}$, S. Pulvirenti ${ }^{20}$, O. Rabyang ${ }^{21}$, F. Raffaelli ${ }^{56}$, N. Randazzo ${ }^{1}$, S. Razzaque ${ }^{50}$, D. Real ${ }^{3}$, S. Reck ${ }^{12}$, G. Riccobene ${ }^{20}$, A. Romanov $^{10}$, A. Rovelli $^{20}$, F. Salesa Greus ${ }^{3}$, D. F. E. Samtleben ${ }^{15,52}$, A. Sánchez Losa ${ }^{35}$, M. Sanguineti ${ }^{10,40}$, A. Santangelo ${ }^{51}$, D. Santonocito ${ }^{20}$, P. Sapienza ${ }^{20}$, J. Schnabel ${ }^{12}$, M. F. Schneider ${ }^{12}$, J. Schumann ${ }^{12}$, H. M. Schutte ${ }^{21}$, J. Seneca ${ }^{15}$, I. Sgura $^{35}$, R. Shanidze ${ }^{47}$, A. Sharma ${ }^{53}$, A. Sinopoulou9 ${ }^{9}$ B. Spisso ${ }^{5,39}$, M. Spurio ${ }^{17,18}$, D. Stavropoulos ${ }^{9}$, S. M. Stellacci ${ }^{5,39}$, M. Taiuti ${ }^{10,40}$, Y. Tayalati ${ }^{16}$, E. Tenllado ${ }^{49}$, H. Thiersen $^{21}$, S. Tingay ${ }^{36}$, V. Tsourapis ${ }^{9}$, E. Tzamariudaki ${ }^{9}$, D. Tzanetatos ${ }^{9}$, V. Van Elewyck ${ }^{14,48}$, G. Vasileiadis ${ }^{43}$, F. Versari ${ }^{17,18}$, D. Vivolo ${ }^{5,28}$, G. de Wasseige ${ }^{14}$, J. Wilms ${ }^{54}$, R. Wojaczyński ${ }^{46}$, E. de Wolf ${ }^{15,26}$, T. Yousfi ${ }^{23}$, S. Zavatarelli ${ }^{10}$, A. Zegarelli ${ }^{7,29}$, D. Zito ${ }^{20}$, J. D. Zornoza ${ }^{3}$, J. Zúñiga ${ }^{3}$, N. Zywucka ${ }^{21}$

\footnotetext{
${ }^{1}$ INFN, Sezione di Catania, Via Santa Sofia 64, 95123 Catania, Italy

${ }^{2}$ Université de Strasbourg, CNRS, IPHC UMR 7178, 67000 Strasbourg, France

${ }^{3}$ IFIC-Instituto de Física Corpuscular (CSIC-Universitat de València), c/Catedrático José Beltrán, 2, 46980 Paterna, Valencia, Spain

4 Aix Marseille Univ, CNRS/IN2P3, CPPM, Marseille, France

5 INFN, Sezione di Napoli, Complesso Universitario di Monte S. Angelo, Via Cintia ed. G, 80126 Naples, Italy

${ }^{6}$ Università di Napoli “Federico II”, Dip. Scienze Fisiche "E. Pancini”, Complesso Universitario di Monte S. Angelo, Via Cintia ed. G, 80126 Naples, Italy

7 INFN, Sezione di Roma, Piazzale Aldo Moro 2, 00185 Rome, Italy

${ }^{8}$ Laboratori d'Aplicacions Bioacústiques, Centre Tecnològic de Vilanova i la Geltrú, Universitat Politècnica de Catalunya, Avda. Rambla Exposició, s/n, 08800 Vilanova i la Geltrú, Spain
} 
${ }^{9}$ Institute of Nuclear and Particle Physics, NCSR Demokritos, Ag. Paraskevi Attikis, 15310 Athens, Greece

10 INFN, Sezione di Genova, Via Dodecaneso 33, 16146 Genoa, Italy

${ }^{11}$ Department of Computer Architecture and Technology/CITIC, University of Granada, 18071 Granada, Spain

${ }^{12}$ Friedrich-Alexander-Universität Erlangen-Nürnberg, Erlangen Centre for Astroparticle Physics, Erwin-Rommel-Straße 1, 91058 Erlangen, Germany

${ }^{13}$ Instituto de Investigación para la Gestión Integrada de las Zonas Costeras, Universitat Politècnica de València, C/ Paranimf, 1, 46730 Gandia, Spain

14 Astroparticule et Cosmologie, Université de Paris, CNRS, 75013 Paris, France

15 Nikhef, National Institute for Subatomic Physics, PO Box 41882, 1009 DB Amsterdam, The Netherlands

${ }^{16}$ Faculty of Sciences, University Mohammed V in Rabat, 4 av. Ibn Battouta, B.P. 1014, R.P. 10000 Rabat, Morocco

${ }^{17}$ INFN, Sezione di Bologna, v.le C. Berti-Pichat, 6/2, 40127 Bologna, Italy

18 Dipartimento di Fisica e Astronomia, Università di Bologna, v.le C. Berti-Pichat, 6/2, 40127 Bologna, Italy

${ }^{19}$ KVI-CART University of Groningen, Groningen, The Netherlands

${ }^{20}$ Laboratori Nazionali del Sud, INFN, Via S. Sofia 62, 95123 Catania, Italy

${ }^{21}$ Centre for Space Research, North-West University, Private Bag X6001, Potchefstroom 2520, South Africa

${ }^{22}$ Instituto Español de Oceanografía, Unidad Mixta IEO-UPV, C/ Paranimf, 1, 46730 Gandia, Spain

${ }^{23}$ Faculty of Sciences, University Mohammed I, BV Mohammed VI, B.P. 717, R.P. 60000 Oujda, Morocco

24 Dipartimento di Matematica, Università di Salerno e INFN Gruppo Collegato di Salerno, Via Giovanni Paolo II 132, 84084 Fisciano, Italy

25 ISS, Atomistilor 409, 077125 Măgurele, Romania

${ }^{26}$ Institute of Physics/IHEF, University of Amsterdam, PO Box 94216, 1090 GE Amsterdam, The Netherlands

27 Technical Sciences, TNO, PO Box 155, 2600 AD Delft, The Netherlands

${ }^{28}$ Dipartimento di Matematica e Fisica, Università degli Studi della Campania “Luigi Vanvitelli”, viale Lincoln 5, 81100 Caserta, Italy

${ }^{29}$ Dipartimento di Fisica, Università La Sapienza, Piazzale Aldo Moro 2, 00185 Rome, Italy

${ }^{30}$ Dipartimento di Ingegneria dell’Energia Elettrica e dell'Informazione “Guglielmo Marconi”, Università di Bologna, Via dell’Università 50, 47522 Cesena, Italy

${ }^{31}$ Physics Department, Faculty of Science Semlalia, Cadi Ayyad University, Av. My Abdellah, P.O.B. 2390, 40000 Marrakech, Morocco

32 School of Physics, University of the Witwatersrand, Private Bag 3, Wits, Johannesburg 2050, South Africa

33 Dipartimento di Fisica e Astronomia "Ettore Majorana”, Università di Catania, Via Santa Sofia 64, 95123 Catania, Italy

${ }^{34}$ INFN, LNF, Via Enrico Fermi, 40, 00044 Frascati, Italy

35 INFN, Sezione di Bari, Via Amendola 173, 70126 Bari, Italy

${ }^{36}$ International Centre for Radio Astronomy Research, Curtin University, Bentley, WA 6102, Australia

${ }^{37}$ Subatech, IMT Atlantique, IN2P3-CNRS, Université de Nantes, 4 rue Alfred Kastler-La Chantrerie, BP 20722, 44307 Nantes, France

${ }^{38}$ University of Bari, Via Amendola 173, 70126 Bari, Italy

${ }^{39}$ Dipartimento di Fisica, Università di Salerno e INFN Gruppo Collegato di Salerno, Via Giovanni Paolo II 132, 84084 Fisciano, Italy

${ }^{40}$ Università di Genova, Via Dodecaneso 33, 16146 Genoa, Italy

${ }^{41}$ University Würzburg, Emil-Fischer-Straße 31, 97074 Würzburg, Germany

${ }^{42}$ School of Computing, Engineering and Mathematics, Western Sydney University, Locked Bag 1797, Penrith, NSW 2751, Australia

${ }^{43}$ Laboratoire Univers et Particules de Montpellier, Place Eugène Bataillon-CC 72, 34095 Montpellier Cédex 05, France

44 Physics Department, University La Sapienza, Roma, Piazzale Aldo Moro 2, 00185 Rome, Italy

45 NIOZ (Royal Netherlands Institute for Sea Research), PO Box 59, Den Burg, 1790 AB Texel, The Netherlands

46 National Centre for Nuclear Research, 02-093 Warsaw, Poland

47 Department of Physics, Tbilisi State University, 3, Chavchavadze Ave., 0179 Tbilisi, Georgia

${ }^{48}$ Institut Universitaire de France, 1 rue Descartes, 75005 Paris, France

49 Dpto. de Física Teórica y del Cosmos \& C.A.F.P.E., University of Granada, 18071 Granada, Spain

${ }^{50}$ Department Physics, University of Johannesburg, PO Box 524, Auckland Park 2006, South Africa

${ }^{51}$ Institut für Astronomie und Astrophysik, Eberhard Karls Universität Tübingen, Sand 1, 72076 Tübingen, Germany

52 Leiden Institute of Physics, Leiden University, PO Box 9504, 2300 RA Leiden, The Netherlands

53 Dipartimento di Fisica, Università di Pisa, Largo Bruno Pontecorvo 3, 56127 Pisa, Italy

${ }_{54}^{5}$ Friedrich-Alexander-Universität Erlangen-Nürnberg, Remeis Sternwarte, Sternwartstraße 7, 96049 Bamberg, Germany

${ }^{55}$ Université de Strasbourg, Université de Haute Alsace, GRPHE, 34, Rue du Grillenbreit, 68008 Colmar, France

56 INFN, Sezione di Pisa, Largo Bruno Pontecorvo 3, 56127 Pisa, Italy

${ }^{57}$ Institut für Kernphysik, University of Münster, Wilhelm-Klemm-Str. 9, 48149 Münster, Germany

58 Department of Physics and Astronomy, Utrecht University, PO Box 80000, 3508 TA Utrecht, The Netherlands

59 Accademia Navale di Livorno, Viale Italia 72, 57100 Livorno, Italy

${ }^{60}$ INFN, CNAF, v.le C. Berti-Pichat, 6/2, 40127 Bologna, Italy 
Abstract The next generation of water Cherenkov neutrino telescopes in the Mediterranean Sea are under construction offshore France (KM3NeT/ORCA) and Sicily (KM3NeT/ARCA). The KM3NeT/ORCA detector features an energy detection threshold which allows to collect atmospheric neutrinos to study flavour oscillation. This paper reports the KM3NeT/ORCA sensitivity to this phenomenon. The event reconstruction, selection and classification are described. The sensitivity to determine the neutrino mass ordering was evaluated and found to be $4.4 \sigma$ if the true ordering is normal and $2.3 \sigma$ if inverted, after 3 years of data taking. The precision to measure $\Delta m_{32}^{2}$ and $\theta_{23}$ were also estimated and found to be $85.10^{-6} \mathrm{eV}^{2}$ and $\left({ }_{-3.1}^{+1.9}\right)^{\circ}$ for normal neutrino mass ordering and, $75.10^{-6} \mathrm{eV}^{2}$ and $\left({ }_{-7.0}^{+2.0}\right)^{\circ}$ for inverted ordering. Finally, a unitarity test of the leptonic mixing matrix by measuring the rate of tau neutrinos is described. Three years of data taking were found to be sufficient to exclude $\stackrel{\vec{\nu}_{\tau}}{\vec{\nu}}$ event rate variations larger than $20 \%$ at $3 \sigma$ level.

\section{Introduction}

The standard framework of three neutrino flavour eigenstates $\left(v_{e}, v_{\mu}, v_{\tau}\right)$, which are superpositions of the three mass eigenstates $\left(v_{1}, v_{2}, v_{3}\right)$ with masses $\left(m_{1}, m_{2}, m_{3}\right)$, has been established with more than two decades of neutrino oscillation physics research. By convention, $v_{1}$ is the mass eigenstate with the largest $v_{e}$ component, and $v_{3}$ is the one with the smallest. The ordering of the neutrino mass eigenstates is not yet resolved, and it can be either $m_{1}<m_{2}<m_{3}$ ('normal ordering', NO) or $m_{3}<m_{1}<m_{2}$ ('inverted ordering', IO). The question of the neutrino mass ordering (NMO) is one of the main drivers of neutrino oscillation physics.

Neutrino mixing is described by the Pontecorvo-MakiNakagawa-Sakata (PMNS) matrix, $U,[1-3]$ with

$$
v_{\alpha}=\sum_{i=1}^{3} U_{\alpha i} v_{i},
$$

where $\alpha=e, \mu, \tau$ and $i=1,2,3$. Under the assumption that the mixing matrix $U$ is unitary, it is usually parametrised in terms of three mixing angles $\theta_{12}, \theta_{13}$ and $\theta_{23}$, and a CPviolating phase $\delta_{\mathrm{CP}}$ [4]. Neutrino oscillations are sensitive to mass-squared differences $\Delta m_{i j}^{2}=m_{i}^{2}-m_{j}^{2}(i, j=$ $1,2,3)$. From the three neutrino mass eigenstates two independent mass-squared differences can be constructed, which

Deceased: Giorgos Androulakis.

\footnotetext{
a e-mail: steffen.hallmann@fau.de (corresponding author)

b e-mail: jannik.hofestaedt@fau.de (corresponding author)

c e-mail: mathieu.perrin-terrin@cppm.in2p3.fr (corresponding author)
}

we choose as $\Delta m_{12}^{2}$ and $\pm\left|\Delta m_{23}^{2}\right|$, where the sign of the latter is positive for $\mathrm{NO}$ and negative for IO.

Global fits of the available data form a coherent picture and provide values for $\theta_{12}, \theta_{13}, \theta_{23}, \Delta m_{12}^{2}$ and $\left|\Delta m_{23}^{2}\right|$ with few-percent level precision [5-7]. However, some questions remain: the determination of the value of $\delta_{\mathrm{CP}}$, the octant of $\theta_{23}$ (i.e. whether $\theta_{23}$ is greater or smaller than $\pi / 4$ ) and the neutrino mass ordering (i.e. the sign of $\Delta m_{23}^{2}$ ). The current status is that global fits $[8,9]$ indicate a mild preference for NO over IO, second octant of $\theta_{23}$ and $\delta_{\mathrm{CP}} \approx \pi$ to $\frac{3}{2} \pi$. The experiments driving the NMO sensitivity results are $\mathrm{T} 2 \mathrm{~K}$ [10], NOvA [11], MINOS [12], Super-Kamiokande [13] and IceCube/DeepCore [14]. Notably, the hints for NO tend to weaken in the light of combined analyses [8,9] using the latest results from T2K [15] and NOvA [16].

Deriving strong experimental constraints on the unitarity of the $3 \times 3$ PMNS mixing matrix is challenging, as direct observations of $\stackrel{\vec{V}_{\mu}}{\vec{V}_{\mu}} \rightarrow \stackrel{\vec{V}_{\tau}}{ }$ are difficult and the $\tau$ rest-mass suppresses the $\stackrel{\mathfrak{v}_{\tau}}{\vec{v}}$ interaction cross section. Appearance of $\overrightarrow{\mathfrak{v}_{\tau}}$ has been directly observed at the long baseline CNGS neutrino beam by OPERA $[17,18]$. Evidence for $\stackrel{\mathfrak{v}_{\tau}}{\text { appearance }}$ has also been found on a statistical basis in the atmospheric neutrino flux by Super-Kamiokande [19] and IceCube [20]. However, the uncertainty on the normalisation of the $\vec{\nu}_{\tau}$ signal is currently too large to probe the unitarity of the PMNS mixing matrix. Non-unitarity would imply the incompleteness of the $3 \times 3$ flavour paradigm and could point to the existence of additional neutrino flavours. A statistically highly-

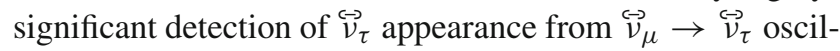
lations of atmospheric neutrinos could make an important contribution to further constrain the PMNS matrix elements involving $\stackrel{\leftrightarrow}{\mathrm{v}_{\tau}}$.

The NMO can be determined by measuring the energy and zenith angle dependent oscillation pattern of few-GeV atmospheric neutrinos that have traversed the Earth [21]. Matterinduced modifications $[22,23]$ of the oscillation probabilities lead to an enhancement of the $\stackrel{\leftrightarrow}{V}_{\mu} \leftrightarrow \stackrel{\leftrightarrow}{v}_{e}$ transition for neutrinos in the case of NO, and anti-neutrinos in the case of IO. Earth matter effects are due to coherent neutrino electron forward scattering. They arise mainly below $E_{v} \lesssim 15 \mathrm{GeV}$ and depend on the electron density of the medium. The largest effects appear around $7 \mathrm{GeV}$ for neutrinos passing through the Earth's mantle and around $3 \mathrm{GeV}$ for neutrinos passing through the Earth's core. The oscillation pattern for neutrinos with respect to anti-neutrinos is flipped between the two mass orderings.

In case of detectors that cannot distinguish between neutrinos and anti-neutrinos on an event-by-event basis, the determination of the NMO can be based on the observation of a net difference in the event rates of atmospheric neutrinos, resulting from a higher interaction cross section (factor $\sim 2$ ) and the existing atmospheric flux difference (factor $\sim 1.1$ ) for neutrinos with respect to anti-neutrinos. Due to this event 
rate difference, the strength of the observed matter effects,

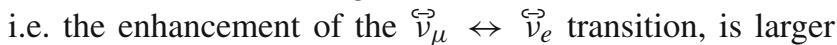
for NO compared to IO. This is the experimental signature exploited by KM3NeT/ORCA and other atmospheric neutrino experiments to determine the NMO.

$\mathrm{KM} 3 \mathrm{NeT}$ is a large research infrastructure that will consist of a network of deep-sea neutrino detectors in the Mediterranean Sea. Two underwater neutrino telescopes, called ARCA and ORCA, are currently under construction [24]. ARCA (Astroparticle Research with Cosmics in the Abyss) is a sparsely instrumented gigaton-scale detector optimised for $\mathrm{TeV}-\mathrm{PeV}$ neutrino astronomy. ORCA (Oscillation Research with Cosmics in the Abyss) is a more densely instrumented detector optimised for measuring the oscillation of few-GeV atmospheric neutrinos in order to determine the neutrino mass ordering.

With atmospheric neutrino data, ORCA can also perform a precise measurement of $\theta_{23}$ and $\Delta m_{23}^{2}$ as well as a highstatistics measurement of $\stackrel{\mathfrak{v}_{\tau}}{\vec{v}}$ appearance in the atmospheric neutrino flux, which allows to probe deviations from the unitarity assumption of the 3-neutrino mixing. Sensitivity for tau-neutrino appearance mainly comes from atmospheric neutrinos with energy $\gtrsim 15 \mathrm{GeV}$ and therefore has only a weak dependence on the still undetermined neutrino mass ordering.

A first estimation of the sensitivity of ORCA to the NMO as well as to other oscillation parameters was published in the 'Letter of Intent for KM3NeT 2.0' (LoI) [24]. Since then, the detector and the analysis methods have been further optimised. First, the detector geometry has been updated. In addition, significant improvements in the neutrino detection efficiency as well as reconstruction performance have been achieved as illustrated in Sect. 2.4. The event classification procedure has been significantly improved as well. We use now three event classes and hit features are included, this is discussed in Sect. 2.5. At the same time the analysis has been refined. The detector response is modeled in greater detail and a more complete list of systematic effects is now considered. These effects partly compensate the expected gain in sensitivity from the improvements mentioned above but make them at the same time more realistic. The updated sensitivities are presented in this paper.

This paper is organised as follows. Section 2 describes the detector design and the simulations performed to obtain the detector response to atmospheric neutrinos, atmospheric muons as well as optical background noise. Then, the algorithms used for event reconstruction and for high flavour purity event classification are described. In Sect. 3, the methods used to analyse these samples and derive the sensitivity to the NMO, the atmospheric oscillation parameters and the $\stackrel{\stackrel{\leftrightarrow}{\nu}_{\tau}}{ }$ appearance are presented together with the results. Finally, Sect. 4 summarises the main detector and analysis updates and the expected sensitivity to neutrino oscillations.

\section{ORCA detector response}

The ORCA detector design comprises a 3-dimensional array of photosensors that register the Cherenkov light produced by relativistic charged particles emerging from neutrinoinduced interactions. The arrival time of the Cherenkov photons and the position of the sensors are used to reconstruct the energy and direction of the incoming neutrino as well as the event topology.

\subsection{Detector design}

The ORCA detector design consists of an array of 115 vertical detection units (DUs) featuring 18 digital optical modules (DOMs) each. Each DOM is a pressure-resistant glass sphere, housing 31 photomultiplier tubes (PMTs) of 3-inch diameter and the related electronics. The KM3NeT PMTs are characterised in [25].

The detector is located at the KM3NeT-France site and the base container of each DU is placed at about $2450 \mathrm{~m}$ depth. The DUs are arranged in a circular footprint with a radius of about $115 \mathrm{~m}$ with an average spacing between the DUs of $20 \mathrm{~m}$. Along a DU, the vertical spacing between the DOMs varies between $8.7 \mathrm{~m}$ to $10.9 \mathrm{~m}$ (due to technical constraints from the deployment procedure) with an average of $9.3 \mathrm{~m}$. The first DOM is at a distance of about $30 \mathrm{~m}$ from the seabed [26]. In total, a volume of about $6.7 \times 10^{6} \mathrm{~m}^{3}$ (equivalent to $7.0 \mathrm{Mt}$ of sea water) is instrumented. This detector configuration is the outcome of an optimisation study using the sensitivity to the NMO as figure of merit.

\subsection{Simulation}

Detailed Monte Carlo (MC) simulations are used to evaluate the detector response to atmospheric neutrinos, atmospheric muons and optical background noise. The simulation chain used for the analysis presented in this paper is similar to the one described in [24].

Neutrino induced interactions in sea water are simulated with gSeaGen [27], a software package based on the widely used GENIE (version 2.12.10) code [28,29]. Neutrinos and antineutrinos in the energy range from 1 to $100 \mathrm{GeV}$ are simulated and weighted to reproduce the conventional atmospheric neutrino flux following the Honda model [30]. All particles emerging from neutrino interactions are propagated with the GEANT4-based software package KM3Sim [31]. Using this software, Cherenkov photons are generated from primary and secondary particles, tracked through the sea water taking into account absorption and scattering, and detected by the PMTs.

Atmospheric muon events are generated using the MUPAGE package [32]. The KM3 package [33,34] is then 
used for tracking the muons in sea water and the subsequent Cherenkov light production.

The PMT response and the readout are simulated using custom KM3NeT software. The digitised PMT output signal is typically called a hit. In this step, the optical background due to Cherenkov light from $\beta$-decays of ${ }^{40} \mathrm{~K}$ in the sea water is also added: an uncorrelated hit rate of $10 \mathrm{kHz}$ per PMT as well as time-correlated noise on multiple PMTs on each DOM $(600 \mathrm{~Hz}$ twofold, $60 \mathrm{~Hz}$ threefold, $7 \mathrm{~Hz}$ fourfold, $0.8 \mathrm{~Hz}$ fivefold and $0.08 \mathrm{~Hz}$ sixfold). The simulated time-correlated noise rate is taken from the data of the first deployed DUs [35]. Finally, the simulated data is filtered by dedicated trigger algorithms to identify events induced by energetic particles. The trigger algorithms are designed to search for large clusters of causally-connected hits. The same trigger algorithms are applied to both simulated and real data.

Compared to the LoI [24], significant improvements have been made in the triggering of faint events with only a few tens of detected photons [36]. A new trigger algorithm has been developed for the needs of ORCA. It is based on only one local coincidence (photons recorded on two or more PMTs of the same DOM within 10ns) and a tunable number of causally-connected single hits on DOMs in the vicinity. A minimum of seven additional hits distributed over at least three different DOMs are required. This new algorithm significantly increases the trigger efficiency in the few-GeV neutrino energy range, while still satisfying the bandwidth requirements of the data acquisition system.

The total trigger rate due to atmospheric muons is about $50 \mathrm{~Hz}$ and noise events add about $54 \mathrm{~Hz}$, while atmospheric neutrinos are triggered with a rate of about $8 \mathrm{mHz}$. In total, 1.4 days of noise events, 14 days of atmospheric muons and more than 15 years of atmospheric neutrinos are simulated. These event samples are sufficient to probe a percent-level background contamination (see Sect. 2.5). In future analysis of real data, the background will be included based on runby-run simulations [34], accounting for the detector and datataking conditions.

\subsection{Event topologies}

Two distinct event topologies can be distinguished in the detector: track-like and shower-like. In the few-GeV energy range, muons are the only particles that can be confidently identified, because they are the only particles that appear as tracks in the detector, with a track length proportional to the muon energy $(\sim 4 \mathrm{~m} / \mathrm{GeV})$. Electrons and hadrons initiate particle showers that develop over distances of a few metres. Compared to elongated muon tracks, these showers appear as localised light sources in the detector. All neutrino-induced events producing a muon with sufficient energy are called

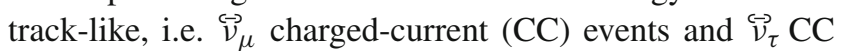

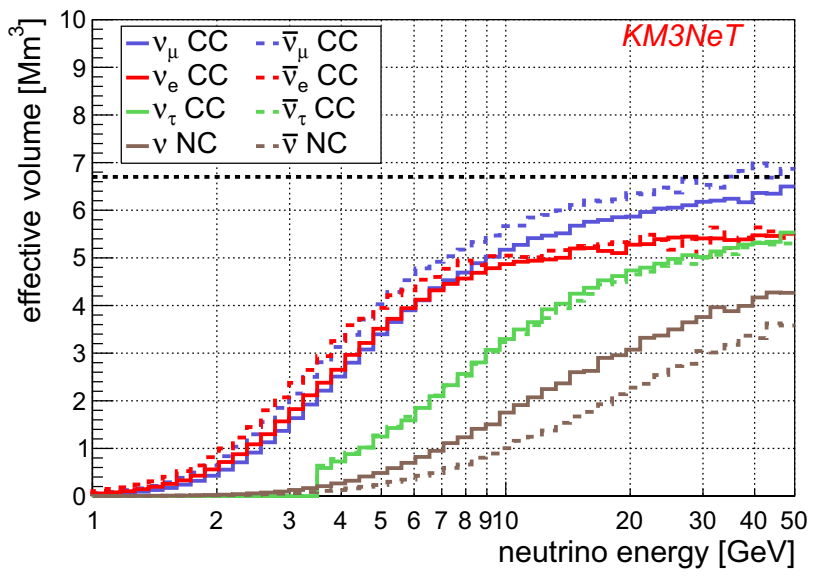

Fig. 1 Effective detector volume as a function of true neutrino energy $E_{v}$ for different neutrino flavours and interactions. Events are weighted according to the Honda atmospheric neutrino flux model and averaged over the zenith angle. Only events reconstructed and selected as upgoing are used. The dashed black line indicates the instrumented volume of the detector

events with muonic $\tau$ decays. All other neutrino-induced events are called shower-like, i.e. $\stackrel{\leftrightarrow}{\vec{v}_{e}, \mu, \tau}$ neutral-current (NC) events, $\stackrel{\leftrightarrow}{V_{e}} \mathrm{CC}$ events and $\stackrel{\leftrightarrow}{\mathcal{V}_{\tau}} \mathrm{CC}$ events with non-muonic $\tau$ decays.

\subsection{Event reconstruction and event selection}

Dedicated reconstruction algorithms are applied for tracklike and shower-like events as well as an event topology classification algorithm. The track and shower reconstruction algorithms are described in [37,38], respectively. Both reconstruction algorithms are maximum likelihood fits and reconstruct the energy and direction as well as interaction vertex position and time. Events reconstructed as upgoing, i.e. with a negative cosine zenith angle, are selected based on the reconstruction quality and containment. The containment criteria are based on the event position and direction inside the instrumented detector volume [36]. The goal of the event preselection is to fulfil two main purposes: suppress background events and select well-reconstructed events with a good reconstruction accuracy.

The effective detector volume after the event preselection is shown in Fig. 1 for upgoing neutrinos weighted according to the Honda atmospheric neutrino flux model [30]. The effective detector volume reaches a plateau and is nearly as large as the instrumented detector volume for $\stackrel{\leftrightarrow}{\mathcal{v}_{\mathrm{e}}, \mu} \mathrm{CC}$ with $E_{v} \gtrsim 15 \mathrm{GeV}$, while $50 \%$ efficiency is reached for $E_{v} \sim 4 \mathrm{GeV}$. Compared to the LoI [24], the turn-on region of the effective detector volume is shifted by about $20 \%$ to lower energies due to improvements in event triggering and reconstruction. Indeed, as discussed in Sect. 2.2, additional methods have been developed to record events with a lower 


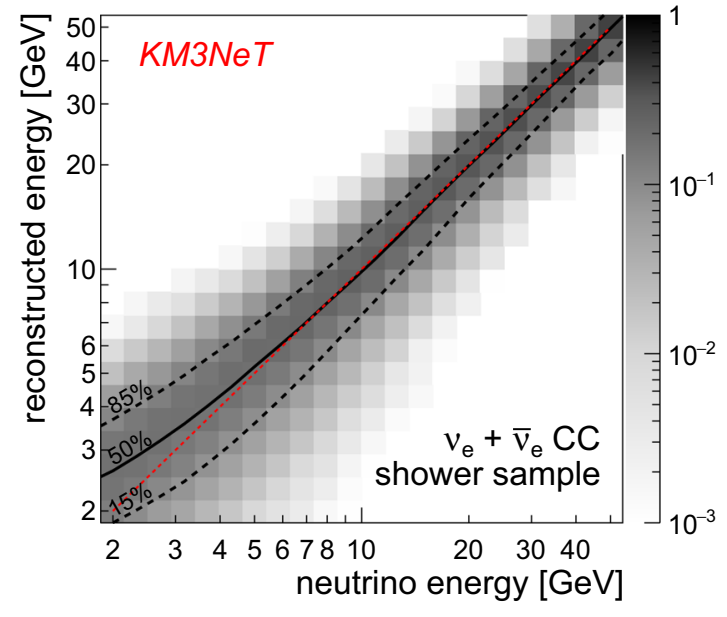

Fig. 2 Probability distribution of the reconstructed energy as a function of true neutrino energy for upgoing $v_{\mathrm{e}} \mathrm{CC}$ and $\bar{v}_{e} \mathrm{CC}$ events classified as shower-like (left) as well as $v_{\mu}$ CC and $\bar{\nu}_{\mu}$ CC events classified as

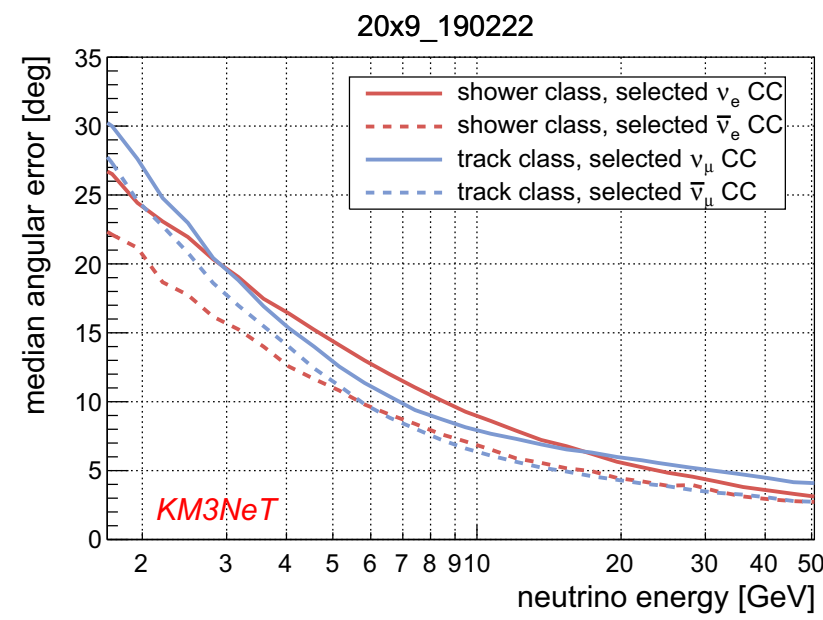

Fig. 3 Median direction resolution as a function of true neutrino energy $E_{v}$ for upgoing $v_{\mathrm{e}} \mathrm{CC}$ and $\bar{v}_{e} \mathrm{CC}$ events classified as shower-like as well as $v_{\mu} \mathrm{CC}$ and $\bar{v}_{\mu} \mathrm{CC}$ events classified as track-like. For a definition of shower- and track-like events see Eq. 2

number of in-time hits from the same DOM but with extra hits causally connected on other DOMs and a similar method is applied at the prefit stage of the reconstruction. These refinements contribute to lower the detection energy threshold. In general, the effective volume is smaller for $\stackrel{\leftrightarrow}{\mathcal{V}} \mathrm{NC}$ and $\stackrel{\leftrightarrow}{\mathfrak{v}_{\tau}} \mathrm{CC}$ than for $\stackrel{\leftrightarrow}{\mathcal{V}_{\mathrm{e}}, \mu} \mathrm{CC}$ events as the outgoing neutrinos are invisible to the detector. For $\bar{v}_{\mathrm{e}, \mu} \mathrm{CC}$ events the effective volume is larger than for $v_{\mathrm{e}, \mu} \mathrm{CC}$ due to the lower average inelasticity and the resulting higher average light yield (at the considered energies hadronic showers have a smaller average light yield than electromagnetic showers). The difference between

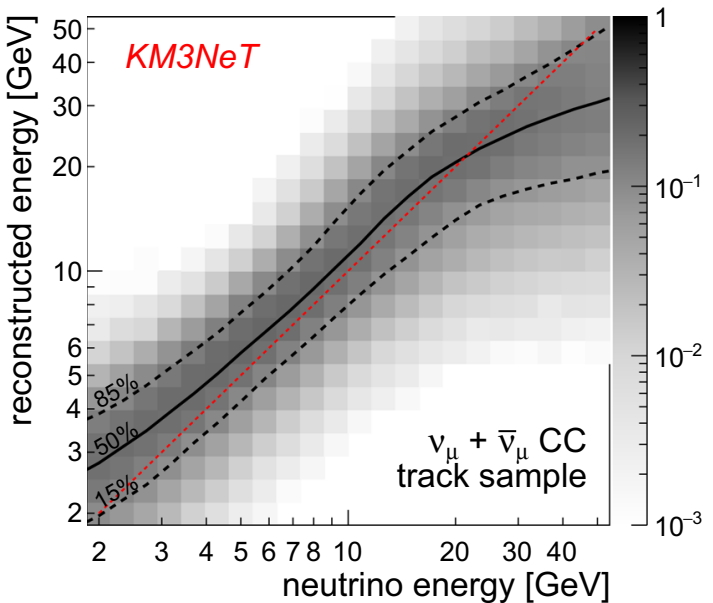

track-like (right). Solid and dashed black lines indicate 50, 15 and 85\% quantiles. For a definition of shower- and track-like events see Eq. 2. The red diagonal line indicates perfect energy reconstruction

$v_{\tau} \mathrm{CC}$ and $\bar{\nu}_{\tau} \mathrm{CC}$ is diluted due to the effect of finite mass of the $\tau$ lepton on the neutrino interaction cross sections [39]. Due to the KM3NeT DOM design, more PMTs are oriented downwards (housed in the lower hemisphere) compared to oriented upwards (housed in the upper hemisphere), resulting in a higher photon detection efficiency for upgoing compared to horizontal events.

In total, a sample of about 66,000 upgoing neutrinos per year, corresponding to a rate of about $2 \mathrm{mHz}$, will be detected and can be used for further analysis. In addition, about $0.4 \mathrm{~Hz}$ of noise events and $0.1 \mathrm{~Hz}$ of atmospheric muon events pass the preselection criteria. To suppress the noise and atmospheric muon background, a more sophisticated event classification is performed, as detailed in Sect. 2.5.

The energy resolution for $\nu_{\mathrm{e}} \mathrm{CC}$ and $\bar{v}_{e} \mathrm{CC}$ events classified as shower-like, as well as $v_{\mu} \mathrm{CC}$ and $\bar{v}_{\mu} \mathrm{CC}$ events classified as track-like are shown in Fig. 2. The energy resolution is Gaussian-like with $\Delta E / E \approx 25 \%$ for $\stackrel{\mathfrak{c}^{\mathrm{v}}}{e} \mathrm{CC}$ events with $E_{v}=10 \mathrm{GeV}$, and it is dominated by the intrinsic light yield fluctuations in the hadronic shower [40]. For $\stackrel{\leftrightarrow}{v}_{\mu} \mathrm{CC}$, the resolution on the neutrino energy levels off at $\Delta E / E \approx 35 \%$ as the reconstructed muon track tends not to be fully contained inside the instrumented volume.

Figure 3 shows the median resolution on the neutrino direction for the same set of simulated neutrino events. At $E_{v}=10 \mathrm{GeV}$, the median neutrino direction resolution is $9.3^{\circ} / 7.0^{\circ} / 8.3^{\circ} / 6.5^{\circ}$ for $v_{\mathrm{e}} / \bar{v}_{e} / v_{\mu} / \bar{v}_{\mu} \mathrm{CC}$ events, respectively. The neutrino direction resolution is dominated by the intrinsic $v$-lepton scattering kinematics [40], resulting in better resolutions for $\bar{v} \mathrm{CC}$ than for $v \mathrm{CC}$ due to the smaller Bjorken-y. 

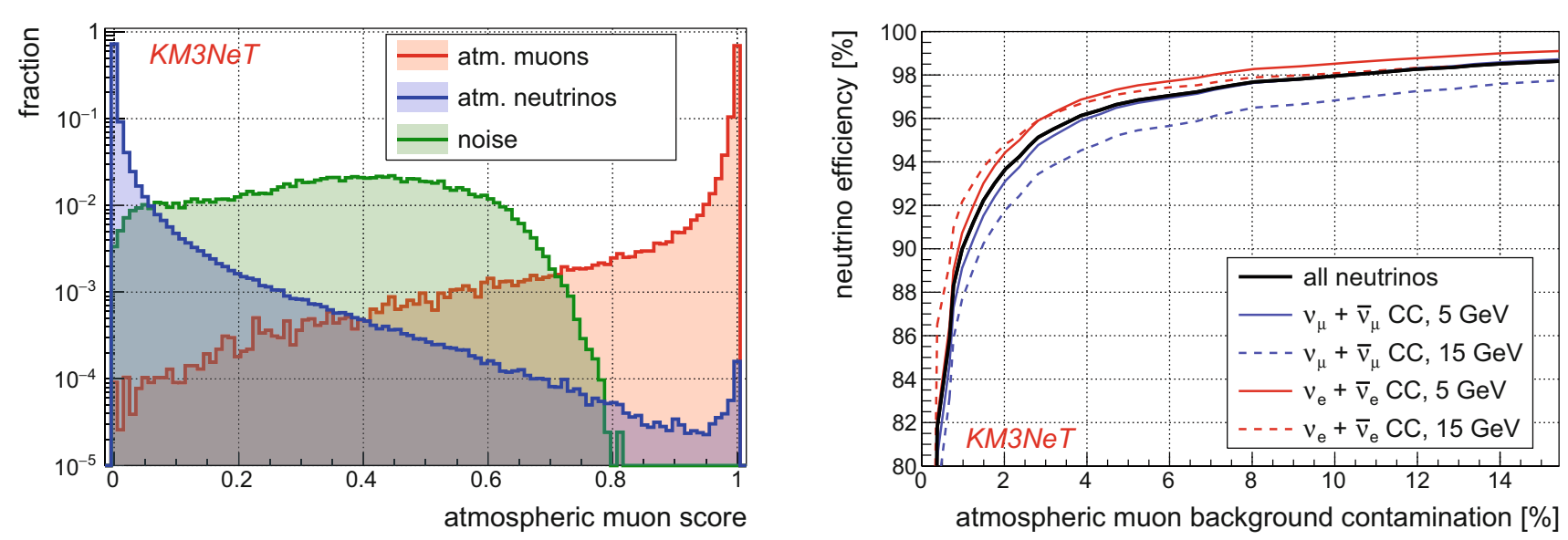

Fig. 4 Left: Distribution of the atmospheric muon score variable for the RDF trained to separate between neutrinos and atmospheric muons, for the main classes of events. Right: Fraction of remaining neutrinos weighted with an oscillated atmospheric flux versus atmospheric muon contamination in the final sample
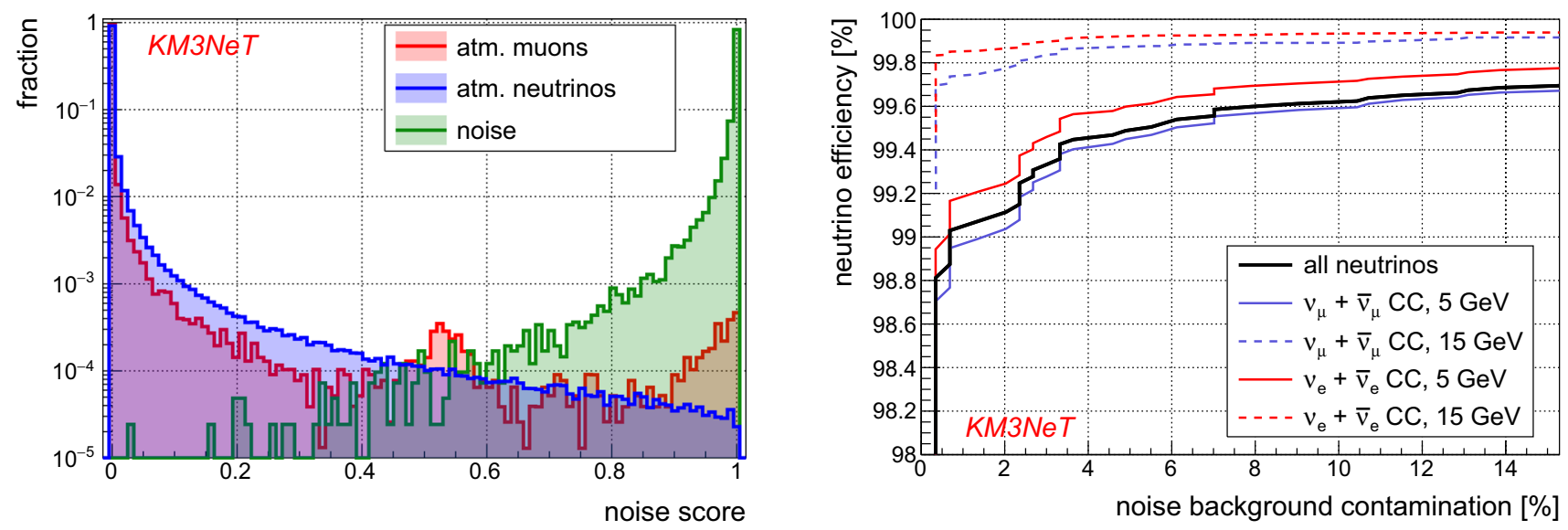

Fig. 5 Left: Distribution of the noise score variable for the RDF aimed to separate between neutrinos and pure noise, for the main classes of events. Right: Fraction of remaining atmospheric neutrinos versus noise event contamination in the final sample

\subsection{Event classification}

For event classification, random decision forests (RDFs) [41] are used, which consist of an ensemble of binary decision trees.

Two RDFs are trained individually for selecting neutrino candidates against each of the two dominant classes of background - atmospheric muons and noise events - and a third one is trained to distinguish track-like from shower-like event topologies.

To train the classifiers, $\stackrel{\leftrightarrow}{\mathfrak{v}_{\mu}} \mathrm{CC}$ events have been used to represent track-like event topologies. For showers $\stackrel{\leftrightarrow}{\mathcal{v}_{e}} \mathrm{CC}$ and $\stackrel{\leftrightarrow}{\mathcal{V}} \mathrm{NC}$ events have been used. The neutrino event distributions were flattened in $\log _{10}$ of neutrino energy and the numbers of events per class were balanced between tracks and showers. In contrast, background was fed with the expected true spectra.
Each trained classifier yields a score variable (atmosph eric_muon_score, noise_score, track_score). These represent the fraction of trees voting for the respective result class. The individual score parameters allow to separately optimise the suppression of the atmospheric muon and noise components using selection cuts and to divide the remaining events into different classes for analysis.

In the training, only events which pass the preselection requirements for either tracks or showers were used. The classifiers were trained independently of each other. Consequently, no further selection based on the resulting score from one of the other classifiers and none of the resulting score variables is used to train the RDFs. In the training, a forest size of 101 trees, ${ }^{1}$ and 50,000 events per class $(25,000$ for noise suppression due to smaller available statistics after

1 The uneven number was chosen for practical purposes only and simplifies consistency of event selection across different analyses ( $>$ vs. $\geq$ ). 

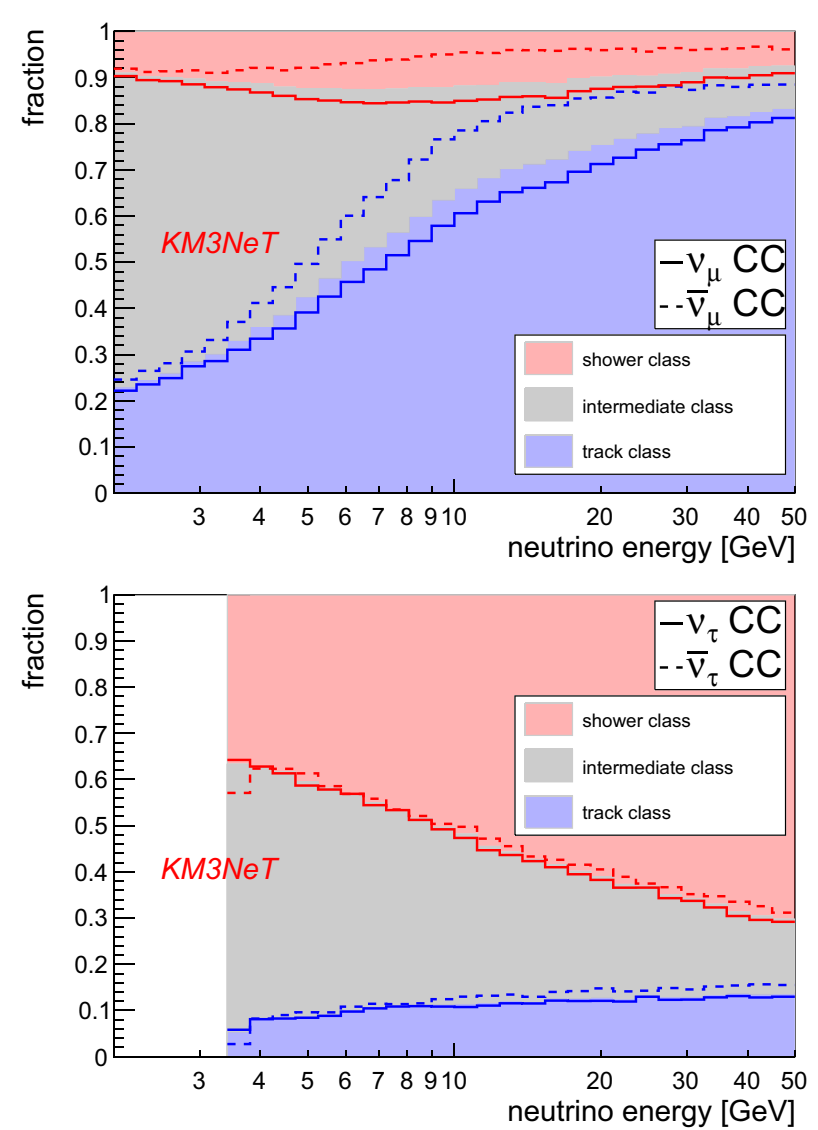

Fig. 6 Fractions of preselected neutrino events of different types that are classified in the track class, the intermediate class, and the shower class, as a function of true neutrino energy. The definition of the classes

preselection) have been used. In the training process, fivefold cross validation was applied.

To ensure diversity of trees within the forest, each tree was trained on a randomly drawn $60 \%$ subset of the training variables and $40 \%$ of the available training events.

The training variables consist of the fitted event parameters and additional variables quantifying the reconstruction quality. These are provided by the track and shower algorithms $[37,38]$. Additional sets of variables fed to the classifier are relative distances between the fitted track and shower hypothesis and variables quantifying how well the Cherenkov light signature is contained within the instrumented volume.

To separate between track- and shower-like signatures, further hit-based variables are added, which have not been used in [24] and exploit the distribution of detected photon hits in the detector. These are based on likelihood ratios of the time and position of the hits expected for the $\vec{v}_{e} \mathrm{CC}$ and $\stackrel{\leftrightarrow}{v_{\mu}}$ CC event hypotheses with respect to the reconstructed position and direction of the shower reconstruction algorithm. More information on the classifier training can be found in [36].
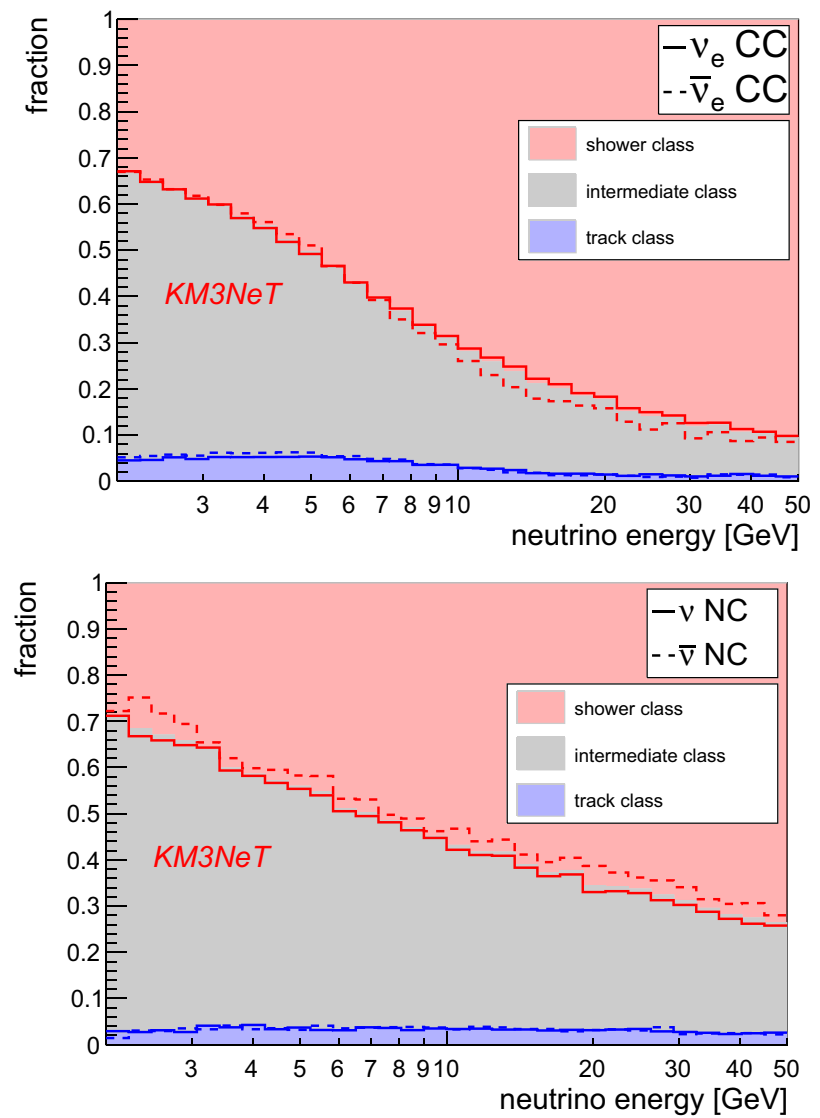

is given in Eq. 2. Coloured areas correspond to the composition of the atmospheric neutrino flux. Solid and dashed lines show individual fractions for neutrinos and anti-neutrinos, respectively

The classifier performance in rejecting the atmospheric muon background is given in Fig. 4. The distribution of the atmospheric_muon_score (left panel) shows a clear separation between neutrinos weighted with an oscillated atmospheric flux and atmospheric muons. The increase of neutrino events with a tracks core $\approx 1$ comes from $\stackrel{\leftrightarrow}{v_{\mu}}$ CC and $\stackrel{\leftrightarrow}{\mathfrak{v}_{\tau}}$ CC events with $\tau^{ \pm}$decay to $\mu^{ \pm}$and is absent for other neutrino channels. Noise events have not been used in training the classifier and therefore are not clustered at the edges of the distributions. A relatively hard cut at atmospheric_muon_score $<0.05$ is used to reach $\mathrm{a} \sim 3 \%$ contamination level, cf. Fig. 4 (right panel). The loss in neutrino efficiency for the atmospheric muon rejection does not strongly depend on the neutrino energy and is about $\sim 5 \%$.

Noise events are rejected sufficiently with a cut on noise_score $<0.1$. As can be seen from Fig. 5 (right panel), the rejection of noise events does not significantly reduce the number of neutrino events in the analysis sample. However, the reduction of neutrino events tends to increase for faint neutrino events with energies 


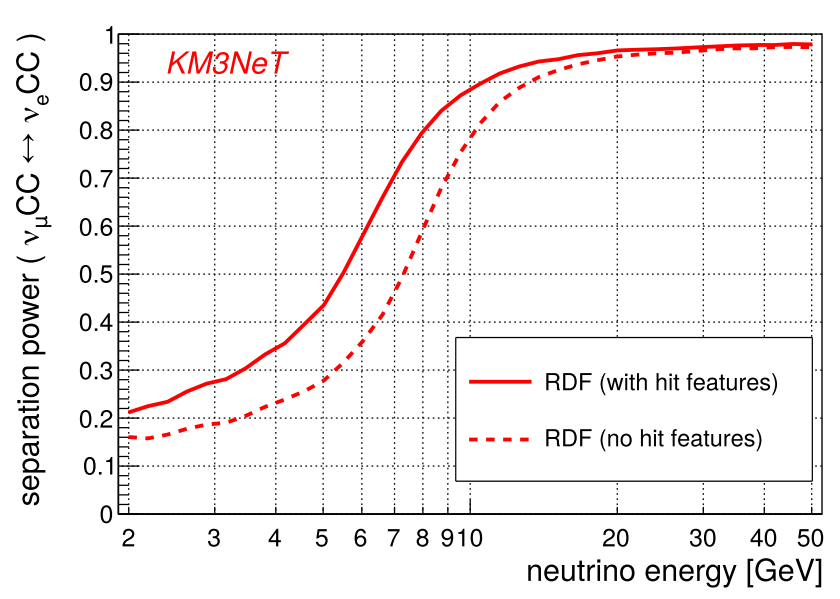

Fig. 7 Comparison of the classifier performance as a function of true neutrino energy in terms of the separation power metric as defined in Eq. 3. Separation power for training with (solid) and without (dashed) hit-based features is shown

near the detection threshold. The proposed cuts on the atmospheric_muon_score and noise_score values reduce the muon and noise contamination of the selected event sample to a level which can be safely neglected in the sensitivity study.

The training of track- versus shower-like neutrino event signatures results in a track_score variable, representing the fraction of trees voting for the candidate event to be tracklike. Using this variable, events can be split in three event classes based on the following criteria:

shower class: passes shower preselection and (track_score $\leq 0.3$ ), intermediate class: passes shower preselection and $(0.3<$ track_score $\leq 0.7)$, track class: passes track preselection and (track_score $>0.7$ ).

The performance of the event type classifier for neutrinos is shown in Fig. 6, where the fractions of events ending up in the respective class are presented as a function of neutrino energy.

The fraction of correctly classified events increases steeply in the energy region up to $\sim 15 \mathrm{GeV}$, where less than $5 \%$ of $\stackrel{\vec{v}}{v_{e}} \mathrm{CC}$ and $\stackrel{\leftrightarrow}{v} \mathrm{NC}$ are mis-classified as tracks. At $\sim 15 \mathrm{GeV}, 85 \% \bar{v}_{\mu} \mathrm{CC}$ and $70 \%$ of $v_{\mu} \mathrm{CC}$ are correctly classified as tracks. The better classification performance for $\bar{v}_{\mu} \mathrm{CC}$ compared to $v_{\mu} \mathrm{CC}$ is due to the different Bjorken-y distribution resulting in longer tracks of the final state muon for $\bar{v}_{\mu} \mathrm{CC}$. The fraction of $\stackrel{\mathrm{C}}{\mathcal{\nu}}_{\tau} \mathrm{CC}$ events classified as tracks is higher compared to $\stackrel{\leftrightarrow}{\nu}_{e} \mathrm{CC}$ and $\stackrel{\leftrightarrow}{\vec{v}} \mathrm{NC}$ reflecting the $17 \%$ branching ratio for muonic tau decays.
To quantify the gain in classification performance when including the additional variables based on the expected hit distributions for $\stackrel{\leftrightarrow}{v}_{\mu} \mathrm{CC}$ and $\stackrel{\leftrightarrow}{v}_{e} \mathrm{CC}$, the separation power, $S$, is used. It quantifies the overlap in the distribution of the track_score between $\stackrel{\mathfrak{v}_{\mu}}{\mathrm{CC}}$ and $\stackrel{\leftrightarrow}{\mathfrak{v}_{e}} \mathrm{CC}$ events by using the correlation coefficient, $C$, and is defined as:

$$
\begin{aligned}
& S(\Delta E)=1-C(\Delta E)
\end{aligned}
$$

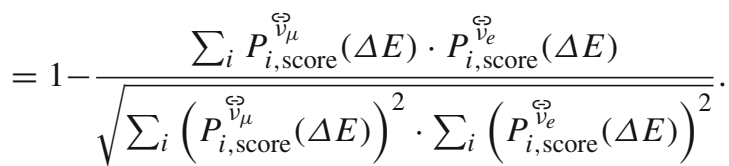

The separation power is calculated in slices of neutrino energy $\Delta E$ by summing over binned probabilities for the track_score values, $P_{i, \text { score }}$. The resulting quantity is shown as a function of neutrino energy in Fig. 7. The event type classification reaches $50 \%$ separation power at $20 \%$ lower neutrino energies when including hit-based variables in the classifier.

\section{Sensitivity calculation}

\subsection{Method}

The neutrino oscillation parameters are studied by analysing the expected bi-dimensional distributions - reconstructed energy, reconstructed cosine zenith angle - of the neutrino candidates in the three event classes (track, intermediate and shower).

These distributions are obtained based on the true energy and cosine zenith angle event distributions split by neutrino interaction type $\left(v_{\mathrm{e}} \mathrm{CC}, \bar{v}_{e} \mathrm{CC}, v_{\mu} \mathrm{CC}, \bar{v}_{\mu} \mathrm{CC}, v_{\tau} \mathrm{CC}, \bar{v}_{\tau} \mathrm{CC}\right.$, $v \mathrm{NC}, \bar{v} \mathrm{NC})$. The true distributions are derived from the neutrino flux [30], the neutrino cross section [42], the probability for each neutrino flavour to oscillate while traversing the Earth computed with the OscProb software [43] and a bi-dimensional parametric description of the detector effective volume. The latter is obtained based on the simulations described in Sect. 2.2.

Each of the eight true energy and cosine zenith angle distributions are then split in the three event classes (track, intermediate and shower), resulting in 24 distributions. The fractions of the distribution classified in each category, given the true neutrino energy, is obtained using parametric functions, derived from simulations.

The distributions of the reconstructed quantities are obtained from these 24 distributions using two sets of parametric functions that describe, first, the probability for a neutrino to be reconstructed at any energy given the true neutrino energy and, second, the probability for a neutrino to be recon- 
structed at any zenith angle given the true neutrino energy and true zenith angle.

These 24 distributions are merged to form the three final distributions of observables (reconstructed energy and cosine zenith angle) for events classified as track, intermediate and shower.

These three final distributions are used as an Asimov data set [44] to derive the median sensitivity to the oscillation parameters under study. A distribution obtained with a given set of oscillation parameters, the null hypothesis, is confronted with other sets, the alternate hypotheses, using $L L_{0}$, the Poisson likelihood $\chi^{2}$ [45], defined as:

$$
\begin{aligned}
L L_{0} & =\sum_{i \in\left[\mathrm{E}^{\mathrm{rec}}, \cos \theta_{\mathrm{z}}^{\mathrm{rec}}\right]} L L_{0, i} \\
& =\sum_{i \in\left[\mathrm{E}^{\mathrm{rec}}, \cos \theta_{\mathrm{z}}^{\mathrm{rec}}\right]}-2.0 \cdot\left(n_{i}^{\text {null }}-n_{i}^{\text {alt }}-n_{i}^{\text {null }} \ln \frac{n_{i}^{\text {null }}}{n_{i}^{\text {alt }}}\right),
\end{aligned}
$$

where $n_{i}^{\text {null }}$ and $n_{i}^{\text {alt }}$ are the expected numbers of events under the null and alternate hypotheses, respectively, in the $i^{\text {th }}$ region of the reconstructed energy - cosine zenith angle plane.

Relevant external information on the neutrino oscillation parameters [6] and model uncertainties are taken into account by adding to $L L_{0}$ extra contributions measuring the discrepancy between the parameter value, $p_{i}^{o b s}$, and the one expected, $p_{i}^{\exp }$, in standard deviation unit, $\sigma_{i}$ :

$L L_{\mathrm{eff}}=L L_{0}+\sum_{i \in \text { parameters }} \frac{\left(p_{i}^{\mathrm{exp}}-p_{i}^{\mathrm{obs}}\right)^{2}}{\sigma_{i}^{2}}$.

The sensitivity to the parameters under study (described in the next sections) is obtained from the $L L_{\mathrm{eff}}$, minimised over all remaining parameters, as $\sqrt{L L_{\text {eff,min }}}$.

A first set of model parameters reflecting the current knowledge on the neutrino flux are considered using the uncertainties reported in [46]:

1. the spectral index of the neutrino flux energy distribution is allowed to vary without constraint,

2. the ratio of upgoing to horizontally-going neutrinos,

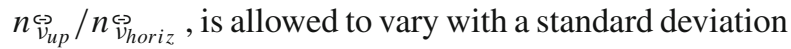
of $2 \%$ of the parameter's nominal value,

3. the ratio between the total number of $\stackrel{\vec{v}}{\vec{v}_{e}}$ and $\stackrel{\stackrel{\leftrightarrow}{v_{\mu}}}{\mu}, n{\stackrel{\leftrightarrow}{v_{e}}}_{e} / n{\stackrel{\leftrightarrow}{v_{\mu}}}_{\mu}$, is allowed to vary with a standard deviation of $2 \%$ of the parameter's nominal value,

4. the ratio between the total number of $v_{\mathrm{e}}$ and $\bar{v}_{e}, n_{v_{\mathrm{e}}} / n_{\bar{v}_{e}}$, is allowed to vary with a standard deviation of $7 \%$ of the parameter's nominal value,
5. the ratio between the total number of $v_{\mu}$ and $\bar{v}_{\mu}, n_{v_{\mu}} / n_{\bar{v}_{\mu}}$, is allowed to vary with a standard deviation of $5 \%$ of the parameter's nominal value.

In addition, two uncertainties on the neutrino cross section are considered:

6. the number of $\mathrm{NC}$ events is scaled by a factor $n_{N C}$ to which no constraint is applied,

7. the number of $\stackrel{\vec{v}_{\tau}}{\mathrm{CC}}$ is scaled by a factor $n_{\tau}^{C C}$ to which no constraint is applied.

Then three uncertainties on the detector response are taken into account:

8. the absolute energy scale of the detector depends on the knowledge of the PMT efficiencies and the water optical properties, as shown in [24] (section 3.4.6). The time dependent PMT efficiencies are monitored permanently with high fidelity, using coincidence signals from ${ }^{40} \mathrm{~K}$ decays, as demonstrated in ANTARES [47]. Several methods are under study to monitor in-situ the water optical properties, exploiting both Cherenkov light from atmospheric muons and ${ }^{40} \mathrm{~K}$ decays as well as signals from artificial light sources. The combination of these methods will allow to constrain the energy scale uncertainty to a few percent. In the study presented here, the energy scale of the detector is allowed to vary with a standard deviation of $5 \%$ around its nominal value,

9. the light yield in hadronic showers, Had. Energy Scale is allowed to vary with a standard deviation of $6 \%$ of the parameter's nominal value, as obtained while comparing two different simulation software packages Gheisha and Fluka [40],

10. the number of events in the three classes is allowed to vary without constraints via three scaling factors $n_{\text {Tracks }}$,

$n_{\text {Intermediate }}, n_{\text {Showers }}$.

Previous studies $[24,48]$ showed that the uncertainty on the Earth model had negligible effects on the NMO sensitivity and is thus ignored in this study. Systematics 2 and 4-10 were not included in the previous analysis [24]. Table 1 reports all the parameters and the external constraints applied to them.

\subsection{NMO sensitivity}

The sensitivity to the neutrino mass ordering is obtained as a function of $\theta_{23}$ using the method described in Sect. 3.1. For every $\theta_{23}$ value, each mass ordering hypothesis - the null hypothesis - is confronted with the reversed one - the alternate hypothesis. The oscillation parameters used for the null hypothesis are reported in Table 2 as well as the constraints applied to them in the minimisation procedure. 
Table 1 Parameter values minimising the $L L$ obtained for 3 years of data taking with NO (IO) as null hypothesis and IO (NO) as alternate hypothesis and using the oscillation parameters from Table 2 . The parameter uncertainties are defined as the values by which the parameter has to vary to increase $L L$ by 1.0. For each parameter value scanned, $L L$ is minimised over the other free parameters

\begin{tabular}{|c|c|c|c|c|}
\hline Parameter & Null hypothesis & Dataset value & Value at Min. & Prior \\
\hline \multirow[t]{2}{*}{$\Delta m_{32}^{2}\left[\mathrm{eV}^{2}\right]$} & NO & $2.528 \times 10^{-3}$ & $\left(2.51_{-0.11}^{+0.11}\right) \times 10^{-3}$ & \multirow[t]{2}{*}{ Free } \\
\hline & IO & $2.436 \times 10^{-3}$ & $\left(2.43_{-0.08}^{+0.10}\right) \times 10^{-3}$ & \\
\hline \multirow[t]{2}{*}{$\Delta m_{21}^{2}\left[\mathrm{eV}^{2}\right]$} & NO & $7.39 \times 10^{-5}$ & $7.39 \times 10^{-5}$ & \multirow[t]{2}{*}{ Fixed } \\
\hline & IO & & & \\
\hline \multirow[t]{2}{*}{$\delta_{\mathrm{CP}}\left[{ }^{\circ}\right]$} & $\mathrm{NO}$ & 221.0 & $162 \pm 180$ & \multirow[t]{2}{*}{ Free } \\
\hline & IO & 282.0 & $190 \pm 180$ & \\
\hline \multirow[t]{2}{*}{$\theta_{13}\left[{ }^{\circ}\right]$} & $\mathrm{NO}$ & 8.60 & $8.63 \pm 0.40$ & \multirow[t]{2}{*}{0.13} \\
\hline & IO & 8.64 & $8.62 \pm 0.29$ & \\
\hline \multirow[t]{2}{*}{$\theta_{12}\left[{ }^{\circ}\right]$} & $\mathrm{NO}$ & 33.82 & 33.82 & \multirow[t]{2}{*}{ Fixed } \\
\hline & IO & & & \\
\hline \multirow[t]{2}{*}{$\theta_{23}\left[^{\circ}\right]$} & $\mathrm{NO}$ & 48.6 & $49.4_{-3.9}^{+2.3}$ & \multirow[t]{2}{*}{ Free } \\
\hline & IO & 48.8 & $41.5_{-1.9}^{+3.8}$ & \\
\hline \multirow[t]{2}{*}{ Spectral index } & NO & 1.0 & $1.00 \pm 0.02$ & \multirow[t]{2}{*}{ Free } \\
\hline & IO & & $1.01 \pm 0.02$ & \\
\hline \multirow[t]{2}{*}{$n_{v_{\text {up }}} / n_{\mathrm{v}_{\text {horiz }}}$} & $\mathrm{NO}$ & 1.0 & $1.01 \pm 0.01$ & \multirow[t]{2}{*}{0.02} \\
\hline & IO & & $1.00 \pm 0.01$ & \\
\hline \multirow[t]{2}{*}{$n \stackrel{\vec{v}}{e}_{e} / n{\stackrel{\vec{\nu}}{\nu_{\mu}}}_{\mu}$} & NO & 1.0 & $1.02 \pm 0.06$ & \multirow[t]{2}{*}{0.02} \\
\hline & IO & & $1.00_{-0.04}^{+0.05}$ & \\
\hline \multirow[t]{2}{*}{$n_{\nu_{\mathrm{e}}} / n_{\bar{v}_{e}}$} & $\mathrm{NO}$ & 1.0 & $1.02_{-0.21}^{+0.22}$ & \multirow[t]{2}{*}{0.07} \\
\hline & IO & & $1.00 \pm 0.16$ & \\
\hline \multirow[t]{2}{*}{$n_{v_{\mu}} / n_{\bar{v}_{\mu}}$} & NO & 1.0 & $0.98_{-0.14}^{+0.15}$ & \multirow[t]{2}{*}{0.05} \\
\hline & $\mathrm{IO}$ & & $1.00_{-0.11}^{+0.12}$ & \\
\hline \multirow[t]{2}{*}{ Energy scale } & $\mathrm{NO}$ & 1.0 & $1.02 \pm 0.05$ & \multirow[t]{2}{*}{0.06} \\
\hline & IO & & $0.99 \pm 0.04$ & \\
\hline \multirow[t]{2}{*}{ Had. energy scale } & $\mathrm{NO}$ & 1.0 & $0.96_{-0.10}^{+0.13}$ & \multirow[t]{2}{*}{0.05} \\
\hline & IO & & $1.00_{-0.08}^{+0.11}$ & \\
\hline \multirow[t]{2}{*}{$n_{N C}$} & NO & 1.0 & $1.02_{-0.37}^{+0.42}$ & \multirow[t]{2}{*}{ Free } \\
\hline & IO & & $0.89_{-0.28}^{+0.32}$ & \\
\hline$n_{\tau}^{C C}$ & NO & 1.0 & $1.05_{-0.20}^{+0.19}$ & Free \\
\hline & IO & & $1.03_{-0.14}^{+0.13}$ & \\
\hline$n_{\text {Intermediate }}$ & NO & 1.0 & $1.00_{-0.06}^{+0.05}$ & Free \\
\hline & IO & & $1.02 \pm 0.04$ & \\
\hline$n_{\text {Tracks }}$ & $\mathrm{NO}$ & 1.0 & $0.98 \pm 0.04$ & Free \\
\hline & IO & & $1.00 \pm 0.03$ & \\
\hline$n_{\text {Showers }}$ & NO & 1.0 & $1.01_{-0.08}^{+0.09}$ & Free \\
\hline & IO & & $1.03_{-0.06}^{+0.07}$ & \\
\hline
\end{tabular}

The distributions of selected events after 3 years of data taking for the null hypothesis assuming $\mathrm{NO}, n_{i}^{\text {null }}$, obtained with the parametric detector response are shown in Fig. 8 using a $40 \times 40$ grid of energy, equally logarithmically spaced between 2 and $100 \mathrm{GeV}$, and cosine zenith angle equally spaced between 0 and -1 . Around $51 \mathrm{~d} 3$ events are expected for the track-class, $63 \mathrm{~d} 3$ for the intermediate-class and $64 \mathrm{~d} 3$ for the shower-class. Figure 8 shows also the $L L_{0, i, \min }$ obtained confronting these distributions with the alternate hypothesis ones.

The sensitivity to the NMO after 3 years of data taking is reported as a function of $\theta_{23}$ for both NMO in Fig. 9a. Assuming the current best estimates for $\theta_{23}$ (see Table 2), the NMO sensitivity is $4.4 \sigma$ if the true $\mathrm{NMO}$ is $\mathrm{NO}$ and $2.3 \sigma$ if it is IO. 
Table 2 Oscillation parameters values used for different analyses for the null hypothesis and constraints applied during the $L L_{\text {eff }}$ minimisation. The values are taken from [6] except the ones identified by a dagger $(\dagger)$ which are extra $\theta_{23}$ and $\delta_{\mathrm{CP}}$ test points used for the NMO sensitivity

Fig. 8 (Left) Expected event distributions for $\mathrm{NO}$ after 3 years of data taking for events classified as track (top), intermediate (middle), and shower (bottom). (right) Signed binned Poisson likelihood $\chi^{2}$ derived using these distributions and the ones obtained minimising $L L_{\mathrm{eff}}$ with the IO hypothesis. If more events are expected for NO than for IO, the value plotted is $L L_{0, i}$ which, as defined in Eq. 4, is positive. Otherwise, the value plotted is $-L L_{0, i}$

\begin{tabular}{llc}
\hline Parameter & Null hypothesis values & Constraints \\
\hline$\Delta m_{21}^{2}$ & $7.39 \times 10^{-5} \mathrm{eV}^{2}$ & Fixed \\
$\theta_{12}$ & $33.82^{\circ}$ & Fixed \\
$\theta_{13}$ & & \\
$\mathrm{NO}$ & $8.60^{\circ}$ & $\pm 0.13^{\circ}$ \\
$\mathrm{IO}$ & $8.64^{\circ}$ & \\
$\Delta m_{31}^{2}$ & & \\
$\mathrm{NO}$ & $2.528 \times 10^{-3} \mathrm{eV}^{2}$ & \\
$\mathrm{IO}$ & $2.436 \times 10^{-3} \mathrm{eV}^{2}$ & Free \\
$\theta_{23}$ & & \\
$\mathrm{NO}$ & $48.6^{\circ},\left[40^{\circ}-50^{\circ}\right] \dagger$ & \\
$\mathrm{IO}$ & $48.8^{\circ},\left[40^{\circ}-50^{\circ}\right] \dagger$ & Free \\
$\delta_{\mathrm{CP}}$ & $221.0^{\circ}, 0^{\circ} \dagger, 180.0^{\circ} \dagger$ & \\
$\mathrm{NO}$ & $282.0^{\circ}, 0^{\circ} \dagger, 180.0^{\circ} \dagger$ & \\
$\mathrm{IO}$ &
\end{tabular}
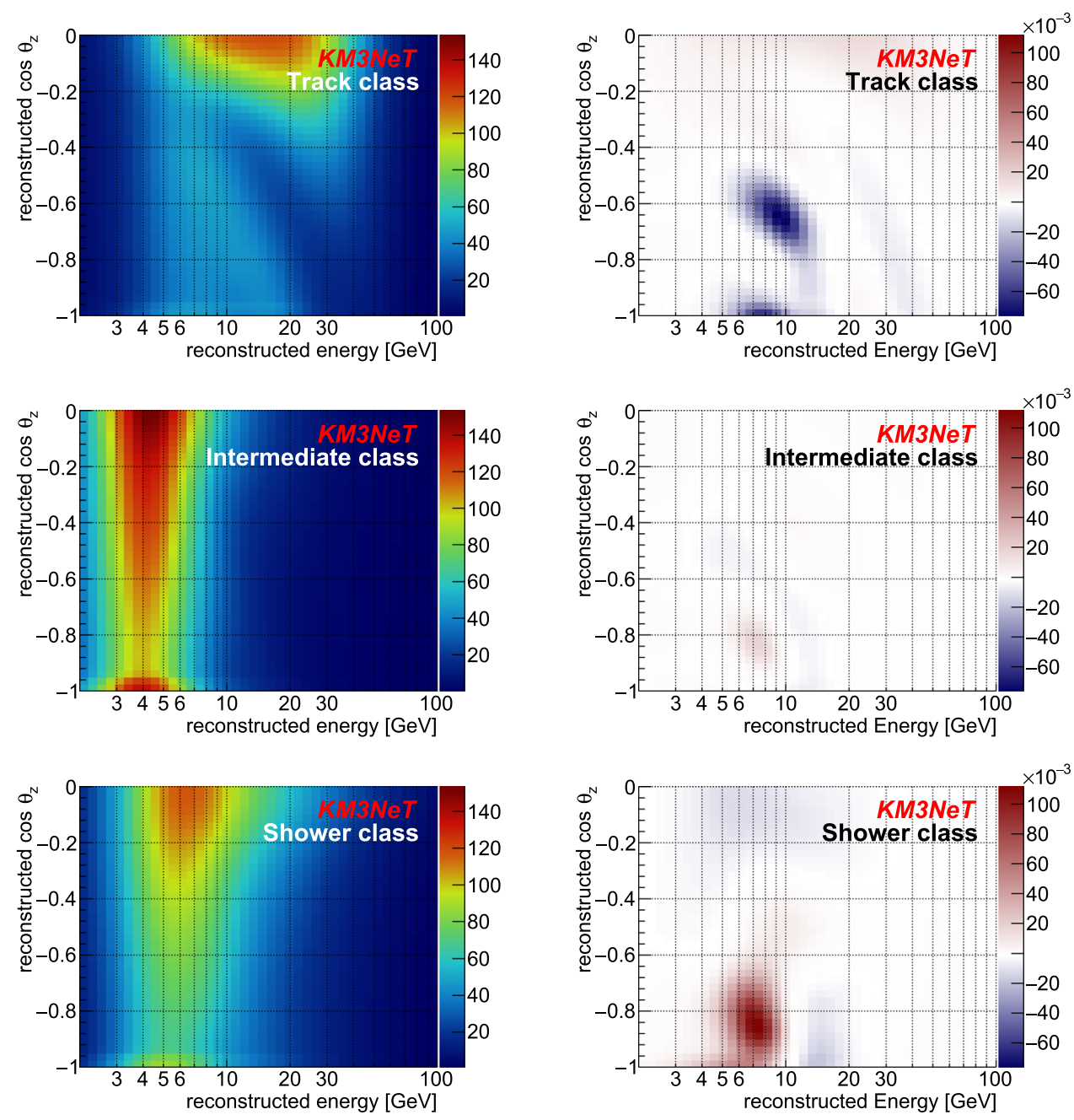


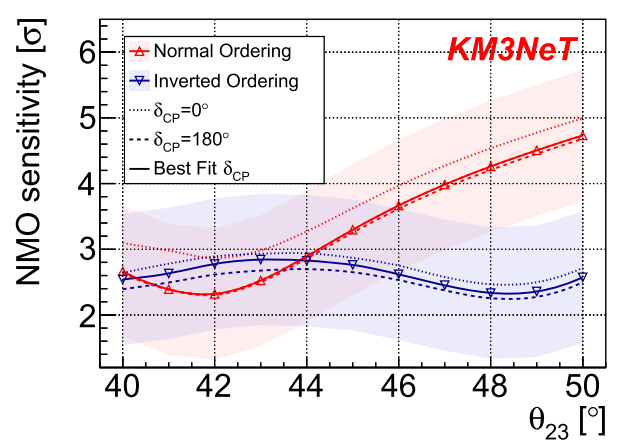

(a)

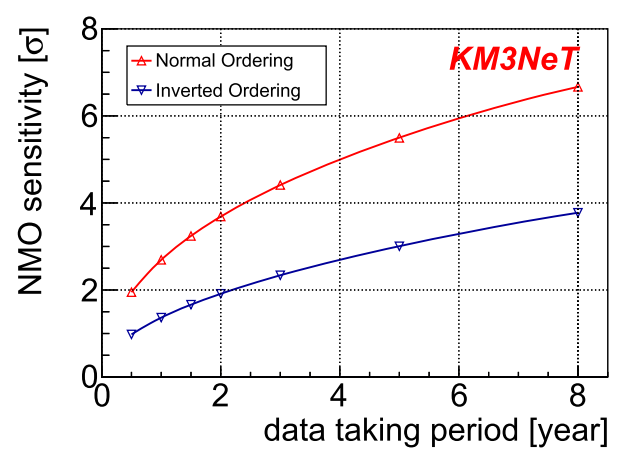

(b)

Fig. 9 a Sensitivity to NMO after 3 years of data taking, as a function of the true $\theta_{23}$ value, for both normal (red upward pointing triangles) and inverted ordering (blue downward pointing triangles) under three assumptions for the $\delta_{\mathrm{CP}}$ value: the world best fit point for $\mathrm{NO}$, IO reported in Table 2 (plain line), $0^{\circ}$ (dotted line) or $180^{\circ}$ (dashed line). The coloured shaded areas represent the sensitivity that $68 \%$ of the experiment realisation would yield, according to the Asimov approach [44]. b Sensitivity to NMO as a function of data taking time for both normal (red upward pointing triangles) and inverted ordering (blue downward pointing triangles) and assuming the oscillation parameters reported in Table 2

Table 1 illustrates the fit results at one test point for oscillation parameters reported in Table 2. None of the systematic uncertainties exhibits a strong pull in this wrong-hierarchy fit, demonstrating that degeneracies between the NMO choice and systematic uncertainties are generally small.

Figure $9 \mathrm{~b}$ shows the sensitivity for both $\mathrm{NMO}$ as a function of data taking time. The NMO can be determined at $3 \sigma$ level after 1.3 years if the true NMO is NO, and after 5.0 years if it is IO.

\subsection{Sensitivity to $\Delta m_{32}^{2}$ and $\theta_{23}$}

The sensitivity to $\Delta m_{32}^{2}$ and $\theta_{23}$ is obtained using the method described in Sect. 3.1. The null hypothesis, assuming the latest oscillation parameter values, reported in Table 2, is confronted with a set of alternate hypotheses, one for each point in the $\Delta m_{32}^{2}, \theta_{23}$ plane. The NMO is kept fixed in the $L L_{\text {eff }}$ minimisation. All $\left(\Delta m_{32}^{2}, \theta_{23}\right)$ points for which the result-

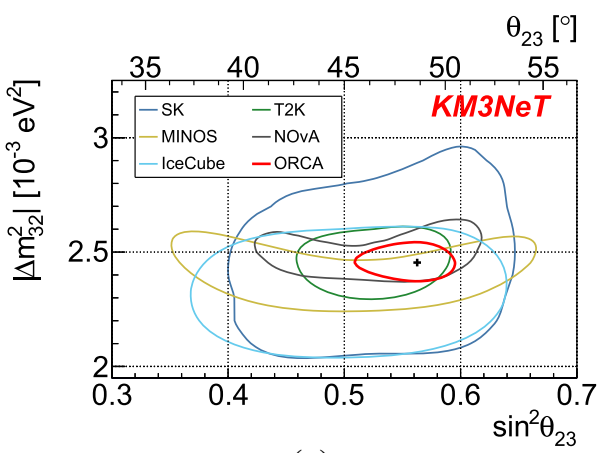

(a)

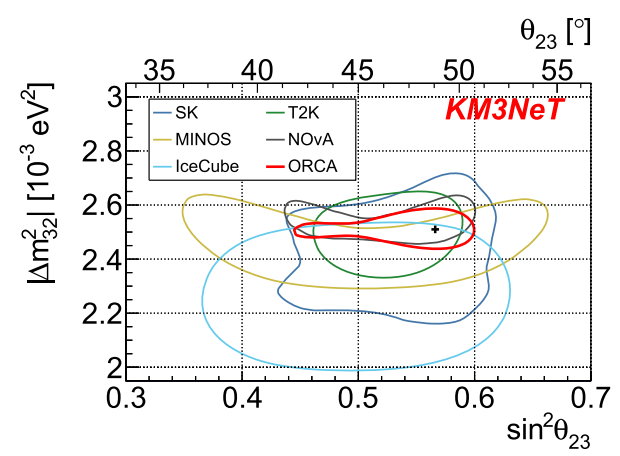

(b)

Fig. 10 Expected measurement precision of $\Delta m_{32}^{2}$ and $\theta_{23}$ for both NO (a) and IO (b) after 3 years of data taking at $90 \%$ confidence level (red) overlaid with results from other experiments [10-14] and the oscillation parameters reported in Table 2 (black cross)

ing $L L_{\text {eff, min }}$ exceeds by 4.61 [4] the $L L_{\text {eff }}$ minimum in the $\left(\Delta m_{32}^{2}, \theta_{23}\right)$ plane are excluded with $90 \%$ confidence level. The oscillation parameters used and the constraints applied during the $L L_{\text {eff }}$ minimisation are reported in Table 2. The resulting $90 \%$ confidence level contours for both NMO are shown in Fig. 10. The $90 \%$ confidence level interval on $\Delta m_{32}^{2}$ and $\theta_{23}$ are $85.10^{-6} \mathrm{eV}^{2}$ and $\left({ }_{-3.1}^{+1.9}\right)^{\circ}$ for $\mathrm{NO}$ and, $75.10^{-6} \mathrm{eV}^{2}$ and $\left({ }_{-7.0}^{+2.0}\right)^{\circ}$ for IO.

The same analysis allows to calculate the significance to determine the octant of $\theta_{23}$. The alternate hypothesis is now the minimal $L L_{\text {eff }}$ for $\theta_{23}$ in the opposite octant with respect to the true $\theta_{23}$ value. The results are shown in Fig. 11, which illustrates the needed data taking time to reach a 1, 2 and $3 \sigma$ octant significance as a function of the true value of $\theta_{23}$. Dashed lines ignore the NMO, while for solid lines the NMO is assumed to be known. KM3NeT/ORCA can constrain the octant with better than $95 \%$ confidence level after 6 years of data taking for $\left|\sin ^{2} \theta_{23}-0.5\right|<0.05$.

\subsection{Sensitivity to ${\stackrel{\leftrightarrow}{V_{\tau}}}_{\tau}$ appearance}

The appearance of $\stackrel{\leftrightarrow}{\mathcal{V}}_{\tau}$ is determined by measuring the normalisation factor $n_{\hat{\nu}_{\tau}}^{c_{\tau}}$ of the $\stackrel{\mathfrak{v}_{\tau}}{\hat{v}_{\tau}}$ contribution. For this study, $\mathrm{NO}$ is assumed. As in the analyses above, the oscillation 


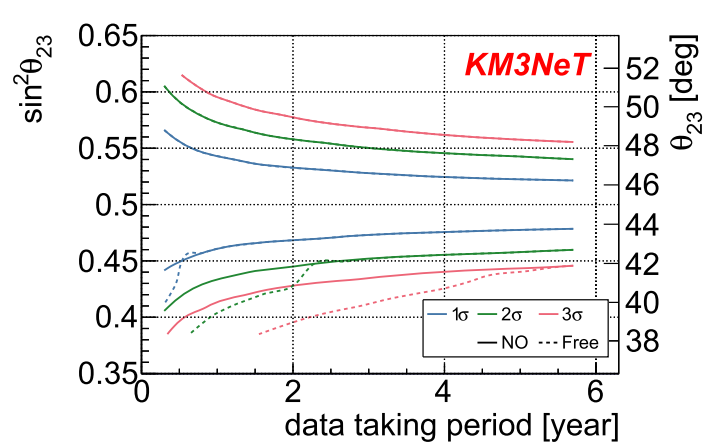

(a)

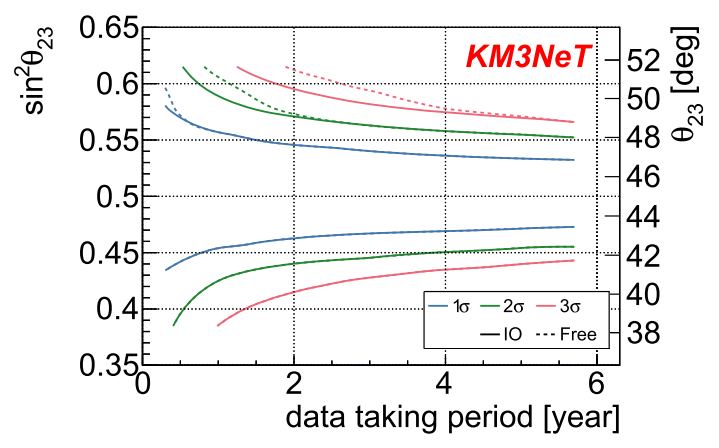

(b)

Fig. 11 Expected sensitivity to determine the $\theta_{23}$ octant at 1 (blue), 2 (green) or $3 \sigma$ (red) as a function of data taking time for both NO (a) and IO (b) assuming the true NMO is known (solid line) or unknown (dashed line). The dashed lines differ from the plain ones when the $L L_{\text {eff }}$ minimisation converges to the wrong NMO

parameter values are taken from Table 2 and the normalisation is fixed to $n_{v_{\tau}} \equiv 1$ for the null hypothesis. The latter is expected if the commonly accepted picture of unitary $3 \times 3$ neutrino mixing is complete and, in addition, the assumed standard model cross sections are correct. A measurement in tension with $n_{\nu_{\tau}}^{\varphi_{\tau}} \equiv 1$ would therefore provide a modelindependent test for new physics. Two choices to scale the $\stackrel{\mathfrak{v}_{\tau}}{\mathcal{V}_{\tau}}$ contribution are possible for the alternate hypotheses. The first is to vary only the $\stackrel{\mathfrak{v}_{\tau}}{\mathrm{V}}$ CC contribution, leaving the $\mathrm{NC}$ contribution fixed to unity. The second allows for a combined $\mathrm{CC}+\mathrm{NC}$ scaling of the $\stackrel{\mathfrak{\nu}_{\tau}}{v_{\tau}}$ flux. Note, that the CC-only case correlates directly with a scaling of the $\vec{\leftrightarrow}_{\tau} \mathrm{CC}$ cross section. Both choices, CC-only and CC+NC normalisation scaling, have been adopted in previous experiments $([18,19]$ and [20], respectively).

The sensitivity is evaluated using the method described in Sect. 3.1 extended by the additional scaling parameter $n_{\nu_{\tau}}$, affecting the ${\stackrel{\leftrightarrow}{\nu_{\tau}}}$ CC flux and in case of CC + NC scaling also the $\mathrm{NC}$ fraction that has oscillated into the ${\stackrel{\mathrm{c}}{\mathcal{V}_{\tau}}}_{\tau}$ channel. While oscillations of the $\mathrm{NC}$ do not need to be considered if the overall flux remains unchanged, this is different for $n_{\nu_{\tau}}^{\mathrm{G}} \neq 1$. In this case the procedure to populate the event distributions is modified and includes the oscillated fractions

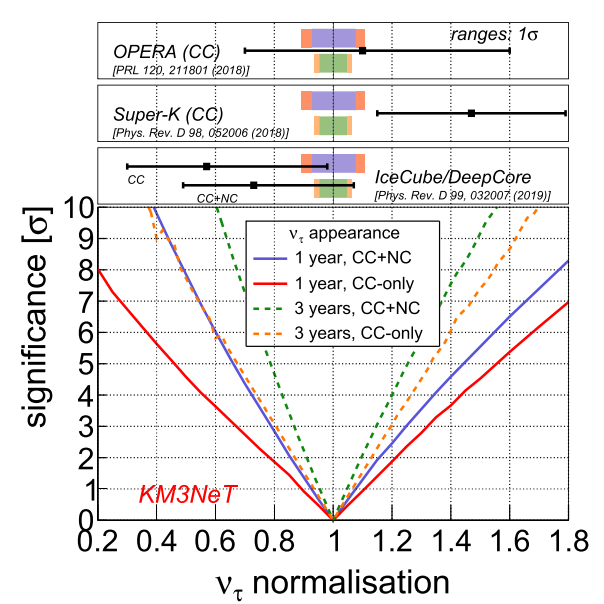

(a)

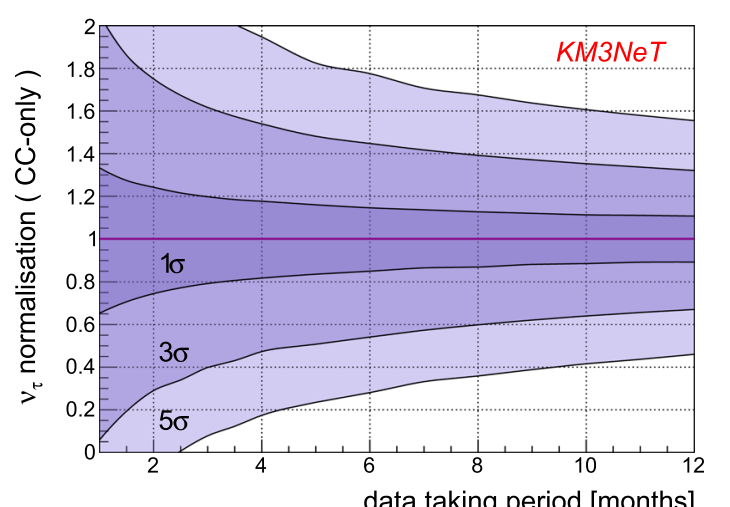

(b)

Fig. 12 Sensitivity to $\stackrel{\leftrightarrow}{\stackrel{V}{V}_{\tau}}$ appearance for $\mathrm{CC}$ and $\mathrm{CC}+\mathrm{NC}$ normalisation scaling after 1 and 3 years of operation (a). Measurements from other experiments [18-20] at $1 \sigma$ level are shown for comparison. In $\mathbf{b}$, $\stackrel{\leftrightarrow}{\mathcal{V}_{\tau}}$ appearance sensitivity for CC scaling is presented as a function of data taking period

of each flavour, which allows to scale the $\stackrel{\mathfrak{v}_{\tau}}{\text { contribution }}$ accordingly.

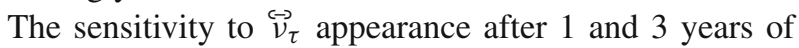
operation for $\mathrm{CC}$ and $\mathrm{CC}+\mathrm{NC}$ normalisation scaling is shown for a scan in $n_{\nu_{\tau}}$ in Fig. 12a. In Fig. 12b, the sensitivity for $\mathrm{CC}$-only scaling is presented as a function of operation time.

KM3NeT/ORCA will already be able to confirm the exclusion of non-appearance with high statistical significance with few months of data-taking. For CC the normalisation can be constrained to $\pm 30 \%$ at $3 \sigma$-level and to $\pm 10 \%$ at $1 \sigma$-level after 1 year of data taking. After 3 years, the normalisation can be constrained to $\pm 20 \%$ at $3 \sigma$-level, and to $\pm 7 \%$ at $1 \sigma$-level. The measured $\stackrel{\leftrightarrow}{\nu}_{\tau}$ normalisation is robust against an incorrectly assumed sign of the still undetermined NMO. This enables KM3NeT/ORCA to measure ${\stackrel{\leftrightarrow}{\nu_{\tau}}}_{\tau}$ appearance already during an early phase of construction [49]. 


\section{Conclusions}

The importance of an independent study of neutrino oscillations, notably the determination of the NMO, has recently been reinforced as earlier hints, which favoured NO, are fading away in the light of latest combined results $[8,9]$.

The KM3NeT/ORCA sensitivity to atmospheric neutrino oscillation has been updated accounting for an optimised detector geometry and major improvements in neutrino trigger and reconstruction algorithms, and data analysis. The trigger algorithm has been improved allowing to more efficiently collect neutrinos in the few-GeV energy range. The algorithms to select neutrino flavour-enriched samples have been optimised using multivariate analysis techniques. Finally, the models used in the statistical analysis have been refined with a realistic description of the systematic uncertainties.

The sensitivity to determine the NMO after 3 years of data taking was found to be $4.4(2.3) \sigma$ if the true NMO is NO (IO) and the other oscillation parameters are set to the current best estimates [6]. The measurement precision on $\Delta m_{32}^{2}$ and $\theta_{23}$ are $85.10^{-6} \mathrm{eV}^{2}$ and $\left({ }_{-3.1}^{+1.9}\right)^{\circ}$ for $\mathrm{NO}$, and $75.10^{-6} \mathrm{eV}^{2}$ and $\left({ }_{-7.0}^{+2.0}\right)^{\circ}$ for IO. Finally, the unitary $3 \times 3$ neutrino mixing paradigm can be assessed by confronting the $\stackrel{\leftrightarrow}{\nu}_{\tau}$ event rate to the expectation in this model. With 3 years of data taking, ${\stackrel{\leftrightarrow}{\nu_{\tau}}}_{\text {event }}$ rate variation larger than $20 \%$ can be excluded at the $3 \sigma$ level.

Acknowledgements The authors acknowledge the financial support of the funding agencies: Agence Nationale de la Recherche (contract ANR-15-CE31-0020), Centre National de la Recherche Scientifique (CNRS), Commission Européenne (FEDER fund and Marie Curie Program), Institut Universitaire de France (IUF), LabEx UnivEarthS (ANR-10-LABX-0023 and ANR-18-IDEX-0001), Paris Île-de-France Region, France; Shota Rustaveli National Science Foundation of Georgia (SRNSFG, FR-18-1268), Georgia; Deutsche Forschungsgemeinschaft (DFG), Germany; The General Secretariat of Research and Technology (GSRT), Greece; Istituto Nazionale di Fisica Nucleare (INFN), Ministero dell'Università e della Ricerca (MIUR), PRIN 2017 program (Grant NAT-NET 2017W4HA7S) Italy; Ministry of Higher Education Scientific Research and Professional Training, ICTP through Grant AF-13, Morocco; Nederlandse organisatie voor Wetenschappelijk Onderzoek (NWO), the Netherlands; The National Science Centre, Poland (2015/18/E/ST2/00758); National Authority for Scientific Research (ANCS), Romania; Ministerio de Ciencia, Innovación, Investigación y Universidades (MCIU): Programa Estatal de Generación de Conocimiento (refs. PGC2018-096663-B-C41, -A-C42, -B-C43, -B-C44) (MCIU/FEDER), Severo Ochoa Centre of Excellence and MultiDark Consolider (MCIU), Junta de Andalucía (ref. SOMM17/6104/UGR), Generalitat Valenciana: Grisolía (ref. GRISOLIA/2018/119) and GenT (ref. CIDEGENT/2018/034 and CIDEGENT/2019/043) programs, La Caixa Foundation (ref. LCF/BQ/IN17/ 11620019), EU: MSC program (ref. 713673), Spain.

Data Availability Statement This manuscript has no associated data or the data will not be deposited. [Authors' comment: This study is based on simulations. The hypotheses used for the simulations are described in the article.]
Open Access This article is licensed under a Creative Commons Attribution 4.0 International License, which permits use, sharing, adaptation, distribution and reproduction in any medium or format, as long as you give appropriate credit to the original author(s) and the source, provide a link to the Creative Commons licence, and indicate if changes were made. The images or other third party material in this article are included in the article's Creative Commons licence, unless indicated otherwise in a credit line to the material. If material is not included in the article's Creative Commons licence and your intended use is not permitted by statutory regulation or exceeds the permitted use, you will need to obtain permission directly from the copyright holder. To view a copy of this licence, visit http://creativecomm ons.org/licenses/by/4.0/.

Funded by $\mathrm{SCOAP}^{3}$.

\section{References}

1. B. Maki, M. Nakagawa, S. Sakata, Prog. Theor. Phys. 28, 870 (1962). https://doi.org/10.1143/PTP.28.870

2. B. Pontecorvo, Sov. Phys. JETP 26, 984 (1968)

3. V. Gribov, B. Pontecorvo, Phys. Lett. B 28, 493 (1969). https://doi. org/10.1016/0370-2693(69)90525-5

4. M. Tanabashi et al. (Particle Data Group), Phys. Rev. D 98(3), 030001 (2018). https://doi.org/10.1103/PhysRevD.98.030001

5. P.F. de Salas, D.V. Forero, C.A. Ternes, M. Tórtola, J.W.F. Valle, Phys. Lett. B 782, 633 (2018). https://doi.org/10.1016/j.physletb. 2018.06.019

6. I. Esteban, M.C. Gonzalez-Garcia, A. Hernandez-Cabezudo, M. Maltoni, T. Schwetz, J. High Energy Phys. 01, 106 (2019). https:// doi.org/10.1007/JHEP01(2019)106

7. F. Capozzi, E. Lisi, A. Marrone, A. Palazzo, Prog. Part. Nucl. Phys. 102, 48 (2018). https://doi.org/10.1016/j.ppnp.2018.05.005

8. I. Esteban, M.C. Gonzalez-Garcia, M. Maltoni, T. Schwetz, A. Zhou, JHEP 09, 178 (2020). https://doi.org/10.1007/ JHEP09(2020) 178

9. K.J. Kelly, P.A. Machado, S.J. Parke, Y.F. Perez Gonzalez, R. Zukanovich-Funchal, Phys. Rev. D 103(1), 013004 (2021). https:// doi.org/10.1103/PhysRevD.103.013004

10. K. Abe et al. (T2K Collaboration), Nature 580(7803), 339 (2020). https://doi.org/10.1038/s41586-020-2177-0

11. M.A. Acero et al. (NOvA Collaboration), Phys. Rev. Lett. 123(15), 151803 (2019). https://doi.org/10.1103/PhysRevLett.123.151803

12. A. Aurisano, Recent results from MINOS and MINOS+, XXVIII International Conference on Neutrino Physics and Astrophysics (2018). https://doi.org/10.5281/zenodo. 1286760

13. K. Abe et al. (Super-Kamiokande Collaboration), Phys. Rev. D 97(7), 072001 (2018). https://doi.org/10.1103/PhysRevD.97. 072001

14. M.G. Aartsen et al. (IceCube Collaboration), Phys. Rev. Lett. 120(7), 071801 (2018). https://doi.org/10.1103/PhysRevLett.120. 071801

15. P. Dunne, Latest neutrino oscillation results from T2K, XXIX International Conference on Neutrino Physics and Astrophysics (2020). https://doi.org/10.5281/zenodo.4154355

16. A. Himmel, New oscillation results from the NOvA experiment. XXIX International Conference on Neutrino Physics and Astrophysics (2020). https://doi.org/10.5281/zenodo.3959581

17. N. Agafonova et al. (OPERA Collaboration), Phys. Rev. Lett. 115(12), 121802 (2015). https://doi.org/10.1103/PhysRevLett. 115.121802

18. N. Agafonova et al. (OPERA Collaboration), Phys. Rev. Lett. 120(21), 211801 (2018). https://doi.org/10.1103/PhysRevLett. 120.211801 [Erratum: Phys. Rev. Lett. 121, 139901 (2018)] 
19. Z. Li et al. (Super-Kamiokande Collaboration), Phys. Rev. D 98(5), 052006 (2018). https://doi.org/10.1103/PhysRevD.98.052006

20. M.G. Aartsen et al. (IceCube Collaboration), Phys. Rev. D 99(3), 032007 (2019). https://doi.org/10.1103/PhysRevD.99.032007

21. E.K. Akhmedov, S. Razzaque, A.Yu. Smirnov, J. High Energy Phys. 02, 82 (2013). https://doi.org/10.1007/JHEP02(2013)082

22. L. Wolfenstein, Phys. Rev. D 17, 2369 (1978). https://doi.org/10. 1103/PhysRevD.17.2369

23. S.P. Mikheyev, A.Y. Smirnov, Sov. J. Nucl. Phys. 42, 913 (1985)

24. S. Adrián-Martínez et al., KM3NeT Collaboration. J. Phys. G 43(8), 084001 (2016). https://doi.org/10.1088/0954-3899/43/8/ 084001

25. S. Aiello et al. (KM3NeT Collaboration), JINST 13(05), P05035 (2018). https://doi.org/10.1088/1748-0221/13/05/P05035

26. S. Aiello et al. (KM3NeT Collaboration), JINST 15(11), P11027 (2020). https://doi.org/10.1088/1748-0221/15/11/P11027

27. S. Aiello (KM3NeT Collaboration), Comput. Phys. Commun. 256, 107477 (2020). https://doi.org/10.1016/j.cpc.2020.107477

28. C. Andreopoulos et al., Nucl. Instrum. Methods A 614, 87 (2010). https://doi.org/10.1016/j.nima.2009.12.009

29. C. Andreopoulos et al., The GENIE neutrino Monte Carlo generator: physics and user manual (2015). arXiv:1510.05494 [hep-ph]

30. M. Honda, M.S. Athar, T. Kajita, K. Kasahara, S. Midorikawa, Phys. Rev. D 92, 023004 (2015). https://doi.org/10.1103/ PhysRevD.92.023004

31. A.G. Tsirigotis, A. Leisos, S.E. Tzamarias, Nucl. Instrum. Methods A 626-627, S185 (2011). https://doi.org/10.1016/j.nima.2010.06. 258

32. G. Carminati, A. Margiotta, M. Spurio, Comput. Phys. Commun. 179, 915 (2008). https://doi.org/10.1016/j.cpc.2008.07.014

33. D. Bailey, Monte Carlo tools and analysis methods for understanding the ANTARES experiment and predicting its sensitivity to dark matter. Ph.D. thesis, University of Oxford (2002)

34. A. Albert et al. (ANTARES Collaboration), JCAP 01, 064 (2021). https://doi.org/10.1088/1475-7516/2021/01/064

35. M. Ageron et al. (KM3NeT Collaboration), Eur. Phys. J. C 80(2), 99 (2020). https://doi.org/10.1140/epjc/s10052-020-7629-z
36. S. Hallmann, Sensitivity to atmospheric tau-neutrino appearance and all-flavour search for neutrinos from the Fermi Bubbles with the deep-sea telescopes KM3NeT/ORCA and ANTARES. Ph.D. thesis, Friedrich-Alexander-Universität Erlangen-Nürnberg (FAU) (2021). https://nbn-resolving.org/urn: nbn:de:bvb:29-opus4-157495

37. L. Quinn, Neutrino mass hierarchy determination with KM3Net/ORCA. Ph.D. thesis, Aix-Marseille University (2018). http://hal.in2p3.fr/tel-02265297

38. J. Hofestädt, Measuring the neutrino mass hierarchy with the future KM3NeT/ORCA detector. Ph.D. thesis, FriedrichAlexander-Universität Erlangen-Nürnberg (FAU) (2017). https:// nbn-resolving.org/urn:nbn:de:bvb:29-opus4-82770

39. Y.S. Jeong, M.H. Reno, Phys. Rev. D 82, 033010 (2010). https:// doi.org/10.1103/PhysRevD.82.033010

40. S. Adrián-Martínez et al. (KM3NeT Collaboration), JHEP 05, 008 (2017). https://doi.org/10.1007/JHEP05(2017)008

41. L. Breiman, Mach. Learn. 45(1), 5 (2001). https://doi.org/10.1023/ A: 1010933404324

42. J.A. Formaggio, G.P. Zeller, Rev. Mod. Phys. 84, 1307 (2012). https://doi.org/10.1103/RevModPhys.84.1307

43. J. Coelho, OscProb neutrino oscillation calculator. https://github. com/joaoabcoelho/OscProb

44. G. Cowan, K. Cranmer, E. Gross, O. Vitells, Eur. Phys. J. C 71, 1554 (2011). https://doi.org/10.1140/epjc/s10052-011-1554-0 [Erratum: Eur. Phys. J. C 73, 2501 (2013)]

45. S. Baker, R.D. Cousins, Nucl. Instrum. Methods 221, 437 (1984). https://doi.org/10.1016/0167-5087(84)90016-4

46. G.D. Barr, T.K. Gaisser, S. Robbins, T. Stanev, Phys. Rev. D 74, 094009 (2006). https://doi.org/10.1103/PhysRevD.74.094009

47. A. Albert et al. (ANTARES Collaboration), Eur. Phys. J. C 78(8), 669 (2018). https://doi.org/10.1140/epjc/s10052-018-6132-2

48. A.M. Dziewonski, D.L. Anderson, Phys. Earth Planet. Inter. 25, 297 (1981). https://doi.org/10.1016/0031-9201(81)90046-7

49. B. Strandberg, S. Hallmann, PoS ICRC2019, 1019 (2019). https:// doi.org/10.22323/1.358.1019 